\title{
NUEVA TEORÍA SOBRE LA DISTRIBUCIÓN DE LOS NÚMEROS PRIMOS
}

\author{
NeW THEORY ABOUT THE PRIME \\ NUMBERS DISTRIBUTION
}

\author{
Luis Alberto Ramírez Castellanos
}

Universidad Nacional Abierta y a Distancia, UNAD

Recibido: 23/04/2017 • Aprobado: 05/24/2017

\section{RESUMEN}

Durante mucho tiempo se ha buscado la explicación a la distribución de los números primos en los números naturales aparentemente aleatoria. A continuación, se desarrolla un hermoso estudio para explicar esta distribución, basados en la definición del concepto de sistema de distribución, con el cual se comprenden las distancias que separan a cada número primo, y en estos sistemas de distribución, estas distancias tiene un patrón que se repite, llamado secuencia de distribución, cuyo valor es igual al primordial. También se explica el defecto de secuencia para no caer en cálculos erróneos en la determinación de números primos. Así, mediante varios teoremas se explican importantes temas, como ejemplo, por qué no todos los números de Euclides de la forma $P_{n} \#+1$ (donde $P_{n}$ \# es el primorial) son primos, y se da otra demostración del postulado de Bertrand. Así, con esta teoría se resuelve el misterio de la distribución, aparentemente caótica, de los números primos, demostrando que siguen un sistema de distribución bien claro. .

Palabras clave: bloque de secuencia, distancias de distribución, defecto de secuencia, factorial, frecuencia de distribución, primordial, producto de fusión, producto de fusión de defecto de secuencia, simetría secuencial, sistemas de distribución secuencia de distribución.

\section{Abstract}

The explanation of the apparently random prime numbers distribution in the natural numbers has been sought for a long time. Below is a beautiful study to explain this distribution, in which each prime number determines a distribution system, the distances that separate each prime number are understood, and in this systems, these distances have a pattern that is repeated, called sequence of distribution, and its value is equal to the primordial. The defect of sequence also is explained to not fall in wrong calculations in the determination of prime numbers; so important themes are explained in various theorems, as an example, why not all Euclid numbers with the form $P_{n} \#+1$ are prime numbers, is explained, and another proof of the Bertran's postulate is given. Thus, with this theory, the apparently chaotic prime numbers distribution is solved, demonstrating that this follows a clearly distribution system.

Key words: block of sequence, distribution defect, distribution distances, distribution frequency, distribution sequence, factorial, fusion product, fusion product of defect sequence, primorial, sequential symmetry. 


\section{Preliminares}

\subsection{Descomposición del factorial}

A continuación en la Tabla 1 se descompone $\mathrm{n}$ ! en factores primos, con $\mathrm{n}$ tomando valores de 1 a 53 , aunque la factorización puede continuar infinitamente para todo valor de :

\section{TABLA 1}

Descomposición del factorial

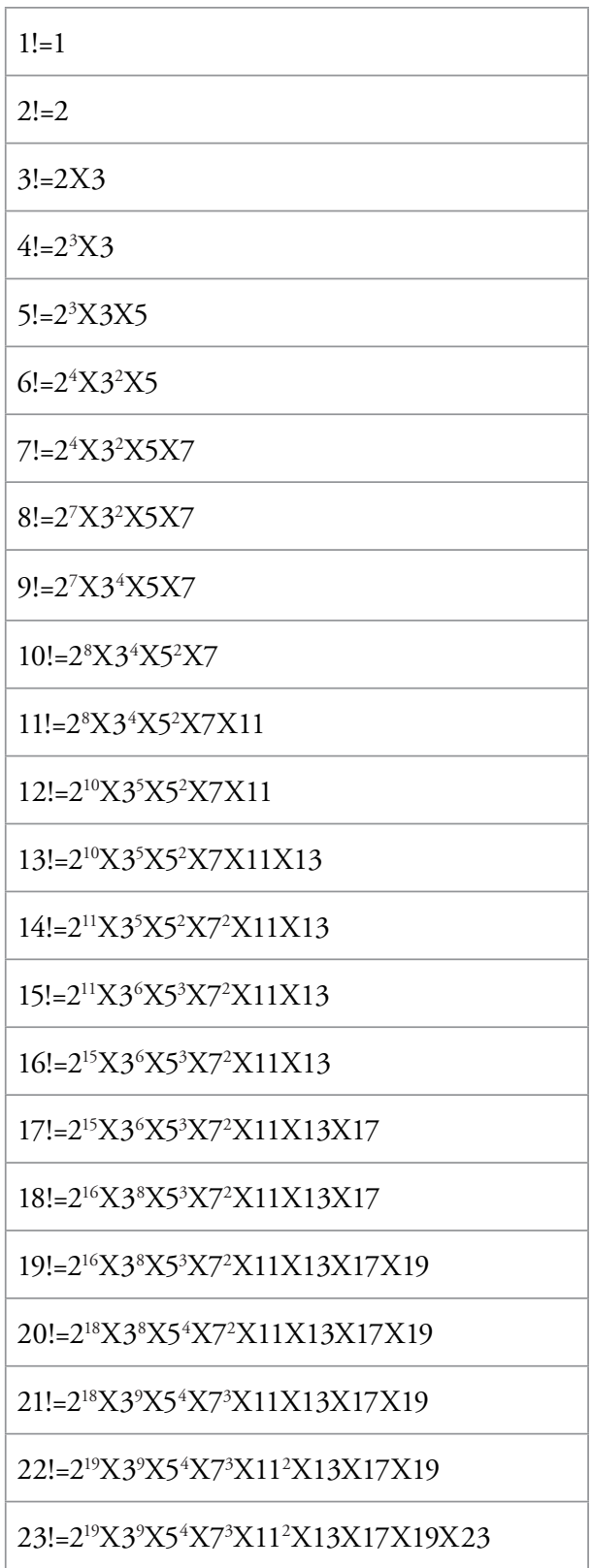

$24 !=2^{22} X 3^{10} X 5^{4} X 7^{3} \mathrm{X} 11^{2} \mathrm{X} 13 \mathrm{X} 17 \mathrm{X} 19 \mathrm{X} 23$

$25 !=2^{22} \mathrm{X} 3^{10} \mathrm{X} 5^{6} \mathrm{X} 7^{3} \mathrm{X} 11^{2} \mathrm{X} 13 \mathrm{X} 17 \mathrm{X} 19 \mathrm{X} 23$

$26 !=2^{23} \times 3^{10} X 5^{6} \times 7^{3} \mathrm{X} 11^{2} \mathrm{X} 13^{2} \mathrm{X} 17 \mathrm{X} 19 \mathrm{X} 23$

$27 !=2^{23} \mathrm{X} 3^{13} \mathrm{X} 5^{6} \mathrm{X} 7^{3} \mathrm{X} 11^{2} \mathrm{X} 13^{2} \mathrm{X} 17 \mathrm{X} 19 \mathrm{X} 23$

$28 !=2^{25} \mathrm{X} 3^{13} \mathrm{X} 5^{6} \mathrm{X} 7^{4} \mathrm{X} 11^{2} \mathrm{X} 13^{2} \mathrm{X} 17 \mathrm{X} 19 \mathrm{X} 23$

$29 !=2^{25} \mathrm{X} 3^{13} \mathrm{X} 5^{6} \mathrm{X} 7^{4} \mathrm{X} 11^{2} \mathrm{X} 13^{2} \mathrm{X} 17 \mathrm{X} 19 \mathrm{X} 23 \mathrm{X} 29$

$30 !=2^{26} \mathrm{X} 3^{14} \mathrm{X} 5^{7} \mathrm{X} 7^{4} \mathrm{X} 11^{2} \mathrm{X} 13^{2} \mathrm{X} 17 \mathrm{X} 19 \mathrm{X} 23 \mathrm{X} 29$

$31 !=2^{26} \mathrm{X} 3^{14} \mathrm{X} 5^{7} \mathrm{X} 7^{4} \mathrm{X} 11^{2} \mathrm{X} 13^{2} \mathrm{X} 17 \mathrm{X} 19 \mathrm{X} 23 \mathrm{X} 29 \mathrm{X} 31$

$32 !=2^{31} X 3^{14} X 5^{7} X 7^{4} X 11^{2} X 13^{2} \mathrm{X} 17 \mathrm{X} 19 \mathrm{X} 23 \mathrm{X} 29 \mathrm{X} 31$

$33 !=2^{31} \mathrm{X} 3^{15} \times 5^{7} \mathrm{X} 7^{4} \mathrm{X} 11^{3} \mathrm{X} 13^{2} \mathrm{X} 17 \mathrm{X} 19 \mathrm{X} 23 \mathrm{X} 29 \mathrm{X} 31$

$34 !=2^{32} \times 3^{15} \times 5^{7} \times 7^{4} \times 11^{3} \times 13^{2} \times 17^{2} \mathrm{X} 19 \mathrm{X} 23 \mathrm{X} 29 \mathrm{X} 31$

$35 !=2^{32} \mathrm{X} 3^{15} \times 5^{8} X 7^{5} \mathrm{X} 11^{3} \mathrm{X} 13^{2} \mathrm{X} 17^{2} \mathrm{X} 19 \mathrm{X} 23 \mathrm{X} 29 \mathrm{X} 31$

$36 !=2^{34} X 3^{17} X 5^{8} X 7^{5} X 11^{3} X 13^{2} X 17^{2} X 19 X 23 X 29 X 31$

$37 !=2^{34} X 3^{17} X 5^{8} X 7^{5} X 11^{3} X 13^{2} X 17^{2} \mathrm{X} 19 \mathrm{X} 23 \mathrm{X} 29 \mathrm{X} 3 \mathrm{X} 37$

$38 !=2^{35} \times 3^{17} \times 5^{8} \times 7^{5} X 11^{3} X 13^{2} X 17^{2} \mathrm{X} 19^{2} \mathrm{X} 23 \mathrm{X} 29 \mathrm{X} 3 \mathrm{X} 37$

$39 !=2^{35} \times 3^{18} \times 5^{8} \times 7^{5} \mathrm{X} 11^{3} \mathrm{X} 13^{3} \mathrm{X} 17^{2} \mathrm{X} 19^{2} \mathrm{X} 23 \mathrm{X} 29 \mathrm{X} 31 \mathrm{X} 37$

$40 !=2^{38} \mathrm{X} 3^{18} \times 5^{9} \times 7^{5} \mathrm{X} 11^{3} \mathrm{X} 13^{3} \mathrm{X} 17^{2} \mathrm{X} 19^{2} \mathrm{X} 23 \mathrm{X} 29 \mathrm{X} 3 \mathrm{X} 37$

$41 !=2^{38} \times 3^{18} \times 5^{9} \mathrm{X} 7^{5} \mathrm{X} 11^{3} \mathrm{X} 13^{3} \mathrm{X} 17^{2} \mathrm{X} 19^{2} \mathrm{X} 23 \mathrm{X} 29 \mathrm{X} 3 \mathrm{X} 37 \mathrm{X} 41$

$42 !=2^{39} \times 3^{19} \times 5^{9} X 7^{6} \mathrm{X} 11^{3} \mathrm{X} 13^{3} \mathrm{X} 17^{2} \mathrm{X} 19^{2} \mathrm{X} 23 \mathrm{X} 29 \mathrm{X} 31 \mathrm{X} 37 \mathrm{X} 41$

$43 !=2^{39} \mathrm{X} 3^{19} \mathrm{X} 5^{9} \mathrm{X} 7^{6} \mathrm{X} 11^{3} \mathrm{X} 13^{3} \mathrm{X} 17^{2} \mathrm{X} 19^{2} \mathrm{X} 23 \mathrm{X} 29 \mathrm{X} 31 \mathrm{X} 3 \mathrm{X} 41 \mathrm{X} 43$

$44 !=2^{41} \mathrm{X} 3^{19} \mathrm{X} 5^{9} \mathrm{X} 7^{6} \mathrm{X} 11^{4} \mathrm{X} 13^{3} \mathrm{X} 17^{2} \mathrm{X} 19^{2} \mathrm{X} 23 \mathrm{X} 29 \mathrm{X} 31 \mathrm{X} 3 \mathrm{X} 41 \mathrm{X} 43$

$45 !=2^{41} X 3^{21} X 5^{10} X 7^{6} X 11^{4} X 13^{3} X 17^{2} X 19^{2} \mathrm{X} 23 \mathrm{X} 29 \mathrm{X} 31 \mathrm{X} 37 \mathrm{X} 4 \mathrm{X} 43$

$46 !=2^{42} X 3^{21} X 5^{10} X 7^{6} X 11^{4} X 13^{3} X 17^{2} X 19^{2} \mathrm{X} 23^{2} \mathrm{X} 29 \mathrm{X} 31 \mathrm{X} 37 \mathrm{X} 4 \mathrm{X} 43$

$47 !=2^{42} X 3^{21} X 5^{10} X 7^{6} X 11^{4} X 13^{3} X 17^{2} X 19^{2} X 23^{2} \mathrm{X} 29 \mathrm{X} 31 \mathrm{X} 3 \mathrm{X} 41 \mathrm{X} 43 \mathrm{X} 47$

$48 !=2^{46} \times 3^{22} X 5^{10} X 7^{6} \times 11^{4} X 13^{3} \times 17^{2} X 19^{2} \mathrm{X} 23^{2} \mathrm{X} 29 \mathrm{X} 31 \mathrm{X} 3 \mathrm{X} 41 \mathrm{X} 43 \mathrm{X} 47$

$49 !=2^{46} \times 3^{22} X 5^{10} X 7^{8} X 11^{4} X 13^{3} X 17^{2} X 19^{2} \mathrm{X} 23^{2} \mathrm{X} 29 \mathrm{X} 31 \mathrm{X} 3 \mathrm{X} 41 \mathrm{X} 43 \mathrm{X} 47$

$50 !=2^{47} X 3^{22} X 5^{12} X 7^{8} X 11^{4} X 13^{3} \times 17^{2} X 19^{2} \mathrm{X} 23^{2} \mathrm{X} 29 \mathrm{X} 31 \mathrm{X} 3 \mathrm{X} 41 \mathrm{X} 43 \mathrm{X} 47$

$51 !=2^{47} X 3^{23} \times 5^{12} X 7^{8} X 11^{4} X 13^{3} X 17^{3} X 19^{2} X 23^{2} X 29 X 31 X 37 X 41 X 43 X 47$

$52 !=2^{49} X 3^{23} X 5^{12} X 7^{8} X 11^{4} X 13^{4} X 17^{3} X 19^{2} \mathrm{X} 23^{2} \mathrm{X} 29 \mathrm{X} 31 \mathrm{X} 3 \mathrm{X} 41 \mathrm{X} 43 \mathrm{X} 47$

$53 !=2^{49} \mathrm{X} 3^{23} \mathrm{X} 5^{12} \mathrm{X} 7^{8} \mathrm{X} 11^{4} \mathrm{X} 13^{4} \mathrm{X} 17^{3} \mathrm{X} 19^{2} \mathrm{X} 23^{2} \mathrm{X} 29 \mathrm{X} 31 \mathrm{X} 37 \mathrm{X} 41 \mathrm{X} 43 \mathrm{X} 47 \mathrm{X} 53$ 
Con la descomposición de $\mathrm{n}$ ! en sus factores primos, se puede notar ciertas características en los cambios de los exponentes de cada factor primo, una de ellas es que en la descomposición del factorial aparece un nuevo número primo cada vez que los exponentes de los números primos ya encontrados como factores, coinciden en no cambiar. Esta característica se puede expresar de la siguiente manera:

considérese a

$n !=2^{a_{1}} * 3^{a_{2}} * 5^{a_{3}} * 7^{a_{4}} * 11^{a_{5}} * \ldots * P_{n}^{a_{i}}$

siendo $\quad a_{1}, a_{2}, a_{3}, \ldots, a_{i} \in N a_{1}, a_{2}, a_{3}, \ldots, a_{i} \in N^{\prime}$ (con $i=1,2, \ldots, N i=1,2, \ldots, N$ ) los exponentes de los factores primos del factorial $n$ !, y si en $(n+1) !(n+1)$ !, ninguno de estos exponentes cambia, entonces aparece un nuevo número primo en su descomposición, de manera que $(n+1) !=2^{a_{1}} * 3^{a_{2}} * 5^{a_{3}} * 7^{a_{4}} * 11^{a_{5}} *$ $\ldots * P_{n}^{a_{i}} * P_{n+1}$

$(n+1) !=2^{a_{1}} * 3^{a_{2}} * 5^{a_{3}} * 7^{a_{4}} * 11^{a_{5}} *$ $\ldots * P_{n}^{a_{i}} * P_{n+1}$

\subsection{Cambio de los exponentes de los factores pri- mos del factorial}

Si se analizan los exponentes de los factores primos, también se observará otra característica importante, y es el hecho de que en los cambios o aumentos de los valores de los exponentes se presentan diferentes distancias.

Teorema 1.2.1. Cada exponente del número primo $P$ se repite cada $P$ veces al ir aumentando el valor de $n$ en $n !$

Demostración. Sea tal $P=P_{n} P=P_{n}$ un factor primo de n!, con exponente $a_{i} a_{i}$, así:

$n !=2^{a_{1}} * 3^{a_{2}} * 5^{a_{3}} * 7^{a_{4}} * 11^{a_{5}} * \ldots * P_{n}^{a_{i}}$,

de manera que

$\left(n+\gamma P_{n}\right) !=2^{a_{1}} * 3^{a_{2}} * 5^{a_{3}} * 7^{a_{4}} * 11^{a_{5}} *$ $\ldots * P_{n}^{\gamma a_{i}}$

$\left(n+\gamma P_{n}\right) !=2^{a_{1}} * 3^{a_{2}} * 5^{a_{3}} * 7^{a_{4}} * 11^{a_{5}} *$

...* $P_{n}^{\gamma a_{i}}$

con $\gamma \in N \gamma \in N$.
Ahora para simplificar los cálculos y eliminar estas repeticiones, al enésimo exponente $\mathrm{n}$ de $\mathrm{P}$ que se desea hallar se le suma un valor $\tau=\tau=0,1,2,3, \ldots \ldots$, (P-1) para que dé como resultado el múltiplo de P siguiente,

para que $\mathrm{P} \mid(n+\tau) n+\tau)$, este resultado se llamará $n_{1}=\frac{n+\tau}{P} n_{1}=\frac{n+\tau}{P}$.

Teorema 1.2.2. La diferencia de los exponentes del factor $P$ de $n$ !, se llama $D$, y aumentan su valor, y aparecen entre $n_{1} n_{1} y\left(n_{1}+1\left(n_{1}+1\right)\right.$, cada $P^{D-1} N=n_{1}$ $P^{D-1} N=n_{1}$, donde $N N$ son los números no múltiplos de $P$.

Demostración. Si $N N$ es múltiplo de P, es porque se aumenta $\mathrm{D}$. sea así $\mathrm{D}=1$, esta distancia aparece cada $N=P^{0} N N=P^{0} N$, y si a $N N$ se le da el valor de un múltiplo de $\mathrm{P}$, de manera que $N=P K N=P K$, entonces $P^{0} P K=P N P^{0} P K=P N$ y se crea $\mathrm{D}=2$ que aparece cada $P^{1} N P^{1} N$, y si a $N N$ se le da el valor de un múltiplo de $\mathrm{P}$, de manera que $P^{1} N=P^{1} P K=P^{2} N$ $P^{1} N=P^{1} P K=P^{2} N$ y se crea $\mathrm{D}=3$, así $\mathrm{D}$ aparece cada $P^{D-1} N P^{D-1} N$.

Teorema 1.2.3. Sea $n_{1}=e n_{1}=e \quad y$ $n_{1}+1=e+D n_{1}+1=e+D$ de $P$, asi el máximo exponente de $P$ en la descomposición del factorial nunca llegará a tener un valor de la forma $e+D_{I} e+D_{I}$, con $1 \leq D_{I}<D 1 \leq D_{I}<D$.

Demostración. Sea ee el máximo exponente de $\mathrm{P}$ en $\mathrm{n}$ !, ahora supóngase que $\mathrm{n}+1=\mathrm{PD}$, de manera que el máximo exponente de $\mathrm{P}$ en $(\mathrm{n}+1)$ ! es $e+D e+D$, ahora sea $f_{1} f_{1}$ el máximo exponente de $\mathrm{P}$ en $(\mathrm{n}-\mathrm{k})$ !, con $1 \leq k<n 1 \leq k<n$, de manera que $f_{1} \leq e f_{1} \leq e$. Ahora sea $f_{2} f_{2}$ el máximo exponente de $\mathrm{P}$ en $(\mathrm{n}+\mathrm{k})$ !, con $\mathrm{k}>1$, de manera que $f_{2} \geq e+D f_{2} \geq e+D$, así que es imposible que exista un exponente máximo para $\mathrm{P}$ de la forma $e+D_{I} e+D_{I}$, con $1 \leq D_{I}<D$ $1 \leq D_{I}<D$.

"Para x real, el símbolo [x] denota el máximo entero menor o igual a x". (Niven \& Zukcerman 1969, 75). 
El siguiente Teorema es similar a: "supóngase que $\mathrm{P}$ denota un número primo. Entonces el mayor exponente e tal que Pe $\mid \mathrm{n}$ ! es $e=\sum_{i=1}^{\infty}\left[\frac{n}{p^{i}}\right] e=\sum_{i=1}^{\infty}\left[\frac{n}{p^{i}}\right]$," (Niven \& Zukcerman 1969, 88)

Teorema 1.2.4. El enésimo exponente $n$ del número primo $P$ en la factorizacion de $n$ ! es: $n=\sum_{D=1}^{j} D V_{D}$

Tal que $V_{j}>0 V_{j}>0{ }_{y} V_{j+1}=0 V_{j+1}=0$.

Donde $D \neq 0 \neq 0$ son las distancias de los exponentes que van tomando el valor de los números naturales 1,2 , $3,4, \ldots \ldots$

$Y$ donde $V D=$

$$
\begin{aligned}
& \left\{\begin{array}{c}
{\left[\frac{n_{1}}{p^{D-1}}\right]-\left[\frac{\left[\frac{n_{1}}{P^{D-1}}\right]}{P}\right] \text { si }\left(\frac{n_{1}}{p^{D-1}}\right) \notin Z} \\
{\left[\frac{n_{1}}{p^{D-1}}\right]-\left[\frac{\left[\frac{n_{1}}{P^{D-1}}\right]}{p}\right]-1 \text { si }\left(\frac{n_{1}}{p^{D-1}}\right) \in(+Z)}
\end{array}\right. \\
& \left\{\begin{array}{c}
{\left[\frac{n_{1}}{p^{D-1}}\right]-\left[\frac{\left[\frac{n_{1}}{P^{D-1}}\right.}{P}\right] \text { si }\left(\frac{n_{1}}{p^{D-1}}\right) \notin Z} \\
{\left[\frac{n_{1}}{p^{D-1}}\right]-\left[\frac{\left[\frac{n_{1}}{P^{D-1}}\right]}{P}\right]-1 \text { si }\left(\frac{n_{1}}{p^{D-1}}\right) \in(+Z)}
\end{array}\right.
\end{aligned}
$$

Demostración. Bien, sea VD las veces que aparece $\mathrm{D}$ en $n_{1} n_{1}$, para saber esto se hace $\left[\frac{n_{1}}{p^{D-1}}\right]\left[\frac{n_{1}}{p^{D-1}}\right]$, pero a este valor hay que quitarle los múltiplos de $\mathrm{P}$ en que no aparece D, así $\left[\frac{n_{1}}{p^{D-1}}\right]-\left[\frac{\left[\frac{n_{1}}{P^{D-1}}\right]}{P}\right]\left[\frac{n_{1}}{p^{D-1}}\right]-\left[\frac{\left[\frac{n_{1}}{p^{D-1}}\right]}{P}\right]$, pero si $\left(\frac{n_{1}}{p^{D-1}}\right)\left(\frac{n_{1}}{p^{D-1}}\right)$ da como resultado un número entero, es porque $n_{1}=K P^{D-1} n_{1}=K P^{D-1}$, entonces a la expresión: $\left[\frac{n_{1}}{p^{D-1}}\right]-\left[\frac{\left[\frac{n_{1}}{P^{D-1}}\right]}{P}\right]\left[\frac{n_{1}}{p^{D-1}}\right]-\left[\frac{\left[\frac{n_{1}}{P^{D-1}}\right]}{P}\right]$ se le resta 1 , ya que las distancias aparecen entre $n_{1} n_{1}$ y $\left(n_{1}+1\left(n_{1}+1\right)\right.$.
A continuación, se multiplican los resultados VD con sus distancias correspondientes, y se suman los resultados entre sí, y esto da el valor del enésimo exponente buscado.

Esto se expresa con la siguiente formula:

$$
n=\sum_{D=1}^{j} D V_{D}
$$

Tal que $V_{j}>0 V_{j}>0$ y $V_{j+1}=0 V_{j+1}=0$.

Ejemplo 1.2.1. Se hallará el $\mathrm{n}=49^{\circ}$ exponente de 2 .

\begin{tabular}{|c|c|c|c|c|c|}
\hline $\mathbf{N}_{1}$ & Exponentes de 2 & \multicolumn{4}{|c|}{ Distancias } \\
\hline 1 & 0 & & & & \\
\hline \multirow[t]{2}{*}{2} & 1 & & & & \\
\hline & & 2 & & & \\
\hline 3 & 3 & & & & \\
\hline \multirow[t]{2}{*}{4} & 4 & & & & \\
\hline & & & 3 & & \\
\hline 5 & 7 & & & & \\
\hline \multirow[t]{2}{*}{6} & 8 & & & & \\
\hline & & 2 & & & \\
\hline 7 & 10 & & & & \\
\hline \multirow[t]{2}{*}{8} & 11 & & & & \\
\hline & & & & 4 & \\
\hline 9 & 15 & & & & \\
\hline \multirow[t]{2}{*}{10} & 16 & & & & \\
\hline & & 2 & & & \\
\hline 11 & 18 & & & & \\
\hline \multirow[t]{2}{*}{12} & 19 & & & & \\
\hline & & & 3 & & \\
\hline 13 & 22 & & & & \\
\hline \multirow[t]{2}{*}{14} & 23 & & & & \\
\hline & & 2 & & & \\
\hline 15 & 25 & & & & \\
\hline \multirow[t]{2}{*}{16} & 26 & & & & \\
\hline & & & & & 5 \\
\hline 17 & 31 & & & & \\
\hline
\end{tabular}

TABla 2

Cambio de los exponentes del número primo dos 
Observando los exponentes en la tabla, en estos se van presentando diferentes distancias: $2,3,4,5, \ldots$

Para no tener en cuenta la repetición de cada exponente, entonces: $\mathrm{n} 1=\left(49^{\circ}+1\right) / 2=25^{\circ}$

Conociendo que el enésimo exponente buscado es el $25^{\circ}$ sin repetición, se utiliza la fórmula (1):

$$
\begin{aligned}
& V_{1}=12 \\
& V_{2}=6 \\
& V_{3}=3 \\
& V_{4}=2 \\
& V_{5}=1 \\
& V_{6}=: 0
\end{aligned}
$$

Como V6 $=0=0$, solo se usaran las distancias 1 , $2,3,4$ y 5 .

Ahora los anteriores resultados se multiplican con sus distancias correspondientes, y los resultados se suman entre sí:

$12^{*} 1=12$

$6^{*} 2=12$

$3^{*} 3=9$

$2 * 4=8$

$1^{*} 5=5$

$12+12+9+8+5=46$ es el $n=49^{\circ}$ exponente de 2 .

\begin{tabular}{|c|c|c|c|c|c|c|c|c|c|}
\hline $\mathbf{N}$ & $P_{1}$ & $\mathbf{P}_{2}$ & $\mathbf{P}_{3}$ & $\mathbf{P}_{4}$ & $\mathbf{P}_{5}$ & $\mathbf{P}_{6}$ & $\mathbf{P}_{7}$ & $P_{8}$ & $P_{9}$ \\
\hline 7 & - & - & - & - & & & & & \\
\hline 8 & $F_{1}$ & - & - & - & & & & & \\
\hline 9 & - & $F_{2}$ & - & - & & & & & \\
\hline 10 & $F_{1}$ & - & $F_{3}$ & - & & & & & \\
\hline 11 & - & - & - & - & - & & & & \\
\hline 12 & $\mathrm{~F}_{1}$ & $F_{2}$ & - & - & - & & & & \\
\hline 13 & - & - & - & - & - & - & & & \\
\hline 14 & $F_{1}$ & - & - & $\mathbf{F}_{4}$ & - & - & & & \\
\hline 15 & - & $\mathbf{F}_{2}$ & $\mathbf{F}_{3}$ & - & - & - & & & \\
\hline 16 & $F_{1}$ & - & - & - & - & - & & & \\
\hline 17 & - & - & - & - & - & - & - & & \\
\hline 18 & $F_{1}$ & $F_{2}$ & - & - & - & - & - & & \\
\hline 19 & - & - & - & - & - & - & - & - & \\
\hline 20 & $F_{1}$ & - & $\mathbf{F}_{3}$ & - & - & - & - & - & \\
\hline 21 & - & $\mathbf{F}_{2}$ & - & $\mathbf{F}_{4}$ & - & - & - & - & \\
\hline 22 & $F_{1}$ & - & - & - & $\mathbf{F}_{5}$ & - & - & - & \\
\hline 23 & - & - & - & - & - & - & - & - & - \\
\hline 24 & $F_{1}$ & $F_{2}$ & - & - & - & - & - & - & - \\
\hline 25 & - & - & $F_{3}$ & - & - & - & - & - & - \\
\hline 26 & $F_{1}$ & - & - & - & - & $F_{6}$ & - & - & - \\
\hline 27 & - & $F_{2}$ & - & - & - & - & - & - & - \\
\hline 28 & $F_{1}$ & - & - & $\mathbf{F}_{4}$ & - & - & - & - & - \\
\hline 29 & - & - & - & - & - & - & - & - & - \\
\hline 30 & $F_{1}$ & $F_{2}$ & $F_{3}$ & - & - & - & - & - & - \\
\hline 31 & - & - & - & - & - & - & - & - & - \\
\hline 32 & $F_{1}$ & - & - & - & - & - & - & - & - \\
\hline 33 & - & $\mathbf{F}_{2}$ & - & - & $\mathbf{F}_{5}$ & - & - & - & - \\
\hline 34 & $\mathrm{~F}_{1}$ & - & - & - & - & - & $\mathbf{F}_{7}$ & - & - \\
\hline 35 & - & - & $\mathbf{F}_{3}$ & $\mathbf{F}_{4}$ & - & - & - & - & - \\
\hline 36 & $F_{1}$ & $F_{2}$ & - & - & - & - & - & - & - \\
\hline
\end{tabular}

\section{Sistemas de distribución}

Considérese la siguiente tabla:

\section{TABLA 3}

Frecuencias de distribución

\begin{tabular}{|c|c|c|c|c|c|c|c|c|c|}
\hline $\mathbf{N}$ & $\mathbf{P}_{1}$ & $\mathbf{P}_{2}$ & $\mathbf{P}_{3}$ & $\mathbf{P}_{4}$ & $\mathbf{P}_{5}$ & $\mathbf{P}_{6}$ & $\mathbf{P}_{7}$ & $\mathbf{P}_{8}$ & $\mathbf{P}_{9}$ \\
\hline $\mathbf{1}$ & & & & & & & & & \\
\hline 2 & - & & & & & & & & \\
\hline 3 & - & - & & & & & & & \\
\hline 4 & $\mathbf{F}_{1}$ & - & & & & & & & \\
\hline 5 & - & - & - & & & & & & \\
\hline 6 & $\mathbf{F}_{1}$ & $\mathbf{F}_{2}$ & - & & & & & & \\
\hline
\end{tabular}




\begin{tabular}{|c|c|c|c|c|c|c|c|c|c|}
\hline $\mathrm{N}$ & $\mathbf{P}_{1}$ & $\mathbf{P}_{2}$ & $\mathbf{P}_{3}$ & $\mathbf{P}_{4}$ & $\mathbf{P}_{5}$ & $\mathbf{P}_{6}$ & $\mathbf{P}_{7}$ & $\mathbf{P}_{8}$ & $\mathbf{P}_{9}$ \\
\hline 37 & - & - & - & - & - & - & - & - & - \\
\hline 38 & $\mathrm{~F}_{1}$ & - & - & - & - & - & - & $\mathrm{F}_{8}$ & - \\
\hline 39 & - & $\mathrm{F}_{2}$ & - & - & - & $\mathrm{F}_{6}$ & - & - & - \\
\hline 40 & $\mathrm{~F}_{1}$ & - & $\mathrm{F}_{3}$ & - & - & - & - & - & - \\
\hline 41 & - & - & - & - & - & - & - & - & - \\
\hline 42 & $\mathrm{~F}_{1}$ & $\mathrm{~F}_{2}$ & - & $\mathrm{F}_{4}$ & - & - & - & - & - \\
\hline 43 & - & - & - & - & - & - & - & - & - \\
\hline 44 & $\mathrm{~F}_{1}$ & - & - & - & $\mathrm{F}_{5}$ & - & - & - & - \\
\hline 45 & - & $\mathrm{F}_{2}$ & $\mathrm{~F}_{3}$ & - & - & - & - & - & - \\
\hline 46 & $\mathrm{~F}_{1}$ & - & - & - & - & - & - & - & $\mathrm{F}_{9}$ \\
\hline
\end{tabular}

La anterior tabla puede continuar indefinidamente horizontal y verticalmente, y los números primos aparecen en las filas coloreadas en azul.

Demostración 2.1. Se llamará frecuencia de distribución Fn de cada primo $P_{n} P_{n}$, a los múltiplos de cada primo, es decir que las frecuencias son distintas para cada primo, asi por ejemplo, la frecuencia $\mathbf{F} \mathbf{1}$ de 2 aparece cada 2 números desde 2, la frecuencia $\mathbf{F} 2$ de 3 aparece cada 3 números a partir de 3, y asi sucesivamente. A medida que se van determinando números primos, se van estableciendo nuevas frecuencias, y la aparición de cada frecuencia determinara los sistemas de distribución.

Demostración 2.2. Se llamará sistemas de distribución $\mathrm{Sn}$ de cada número primo $\mathrm{n}$, a las distancias $\mathrm{Dk}$ secuenciales que determinaran la aparición de los números primos, construidos por las frecuencias de los números primos ya encontrados.

Demostración 2.3. Sea Dk las distancias de distribución de un sistema Sn, cuyos valores son determinados por las diferencias de los números en los que no hay presencia de frecuencias, es decir, los números primos determinados por Sn, de manera que las distancias son las que determinan números primos en $S$..
Anteriormente se observó que en la descomposición del factorial aparecía un nuevo número primo cuando en ningún primo de la descomposición cambiaban sus exponente. Ahora este fenómeno aplica de forma similar en la anterior tabla, en donde aparece un nuevo número primo cuando no aparece ninguna frecuencia en las filas.

A continuación, se construirá la tabla de frecuencias de distribución por partes con el primer número primo, así:

TABLA 4

Frecuencia de $P_{1}=2$

\begin{tabular}{|c|c|}
\hline $\mathbf{N}$ & $P_{1}=2$ \\
\hline 1 & \\
\hline 2 & - \\
\hline 3 & - \\
\hline 4 & $F_{1}$ \\
\hline 5 & - \\
\hline 6 & $F_{1}$ \\
\hline 7 & - \\
\hline 8 & $F_{1}$ \\
\hline 9 & - \\
\hline 10 & $F_{1}$ \\
\hline 11 & - \\
\hline 12 & $F_{1}$ \\
\hline 13 & - \\
\hline 14 & $\mathrm{~F}_{1}$ \\
\hline 15 & - \\
\hline 16 & $F_{1}$ \\
\hline 17 & - \\
\hline 18 & $F_{1}$ \\
\hline 19 & - \\
\hline 20 & $F_{1}$ \\
\hline
\end{tabular}


El primer número primo es $\mathrm{P}_{1}=2$, cuya frecuencia es $\mathrm{F}_{1}=2$ a partir de 2 . Así con el 2 y su frecuencia se construye el primer sistema de distribución S1.

Ahora imagínese que este sistema es el único, de manera que a partir de 2 , los siguientes números primos serán aquellos números que no estén en la frecuencia, los cuales son: 3,5,7,9,11,...., es decir, los números impares, así se construye el primer sistema de distribución, que empieza a funcionar desde el siguiente número primo de 2 , es decir, 3 , que es el primer número que está antes de su primer frecuencia o su primer múltiplo $4, y$ así 3 es el siguiente número primo, esto quiere decir que con $S 1=2-2-2-2-2-2-\ldots$. los siguientes primos aparecerán cada dos números a partir de 3. Así, a partir de tres, los números primos serán: $3+2=5+2=7+2=9+2=11+2=13+2=\ldots \ldots \ldots$ (es evidente que 9 no es un número primo, este error será explicado con la Demostración del defecto de secuencia).

Ahora constrúyase la tabla de frecuencias de distribución con los números primos 2 y 3 :

\section{TABLA 5}

Frecuencias de $\mathrm{P}_{1}=2 \mathrm{Y} \mathrm{P}_{2}=3$

\begin{tabular}{|c|c|c|}
\hline $\mathbf{N}$ & $\mathbf{P}_{1}=\mathbf{2}$ & $\mathbf{P}_{2}=\mathbf{3}$ \\
\hline 1 & & \\
\hline 2 & - & - \\
\hline 3 & - & - \\
\hline 4 & $\mathbf{F}_{1}$ & - \\
\hline 5 & - & $\mathbf{F}_{2}$ \\
\hline 6 & $\mathbf{F}_{1}$ & - \\
\hline 7 & - & - \\
\hline 8 & $\mathbf{F}_{1}$ & $\mathbf{F}_{2}$ \\
\hline 9 & - & - \\
\hline 10 & $\mathbf{F}_{1}$ & - \\
\hline 11 & - & \\
\hline
\end{tabular}

\begin{tabular}{|c|c|c|}
\hline $\mathbf{N}$ & $\mathbf{P}_{\mathbf{1}}=\mathbf{2}$ & $\mathbf{P}_{2}=\mathbf{3}$ \\
\hline 12 & $\mathbf{F}_{1}$ & $\mathbf{F}_{2}$ \\
\hline 13 & - & - \\
\hline 14 & $\mathbf{F}_{1}$ & - \\
\hline 15 & - & $\mathbf{F}_{2}$ \\
\hline 16 & $\mathbf{F}_{1}$ & - \\
\hline 17 & - & - \\
\hline 18 & $\mathbf{F}_{1}$ & $\mathbf{F}_{2}$ \\
\hline 19 & - & - \\
\hline 20 & $\mathbf{F}_{1}$ & - \\
\hline 21 & - & $\mathbf{F}_{2}$ \\
\hline 22 & $\mathbf{F}_{1}$ & - \\
\hline 23 & - & - \\
\hline
\end{tabular}

Aquí aparecen dos frecuencias, $\mathrm{F}_{1}=2$ y $\mathrm{F}_{2}=3$, imagínese que solo existen estas dos frecuencias, y con ellas se construye el segundo sistema de distribución S2 $=2-4-2-4-2-4-2-4-\ldots$ esto quiere decir que con S2, que empieza a funcionar a partir del siguiente número primo del primo hasta donde se construyó el sistema, es decir el siguiente de 3 que es 5, los siguientes números primos aparecerán con las siguientes distancias:2-4-2-4-2-4-2-4-..., así por ejemplo a partir de 5 los números primos siguientes serán: $5+2=7+4=11+2=1$ $3+4=17+2=19+4=23+2=25+4=29+2=\ldots$

Hay que aclarar que los sistemas cambian con cada primo que se encuentra.

Así, como se vio anteriormente, se pueden construir los sistemas S3, S4. S5, S6,...Sn.

Ahora encontrando las distancias presentes en cada sistema, se puede construir un cuadro como el siguiente (el cuadro puede continuar indefinidamente): 
TABLA 6

Cuadro de las distancias de los sistemas de distribución

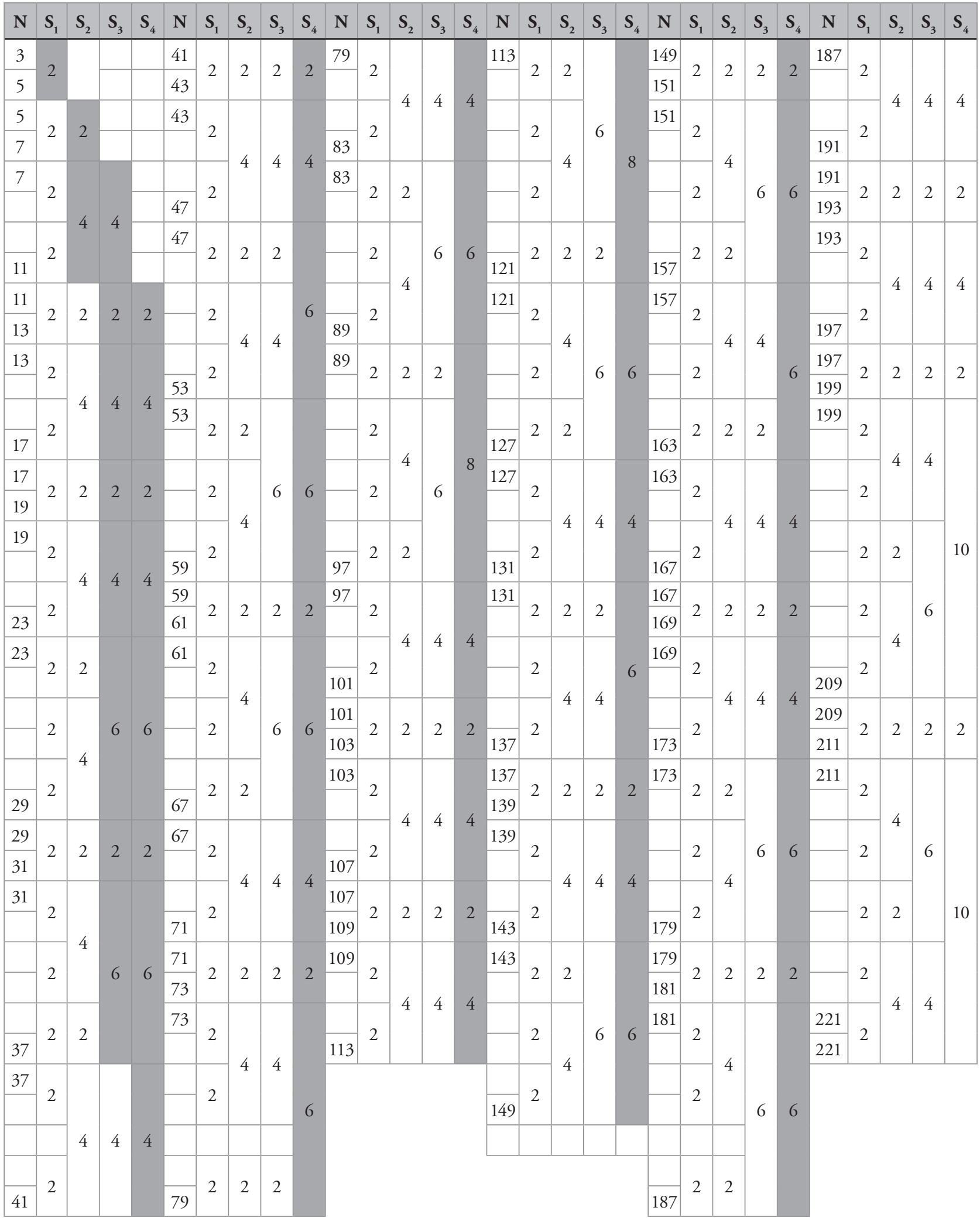


El cuadro anterior solo se hizo hasta $\mathrm{S} 4$ donde $\mathrm{P}_{4}=7$, y como $\mathrm{S}_{4}$ funciona bien hasta el cuadrado de $\mathrm{P}_{5}=11$, es decir que desde 121 empiezan a aparecer también números compuestos como 121, 143, 169, 187, 209, $221, \ldots$, pero si el cuadro de las distancias de los sistemas de distribución se completa hasta $S_{n}$, se perfecciona.

Demostración 2.4. Se llamará secuencia de distribución $\mathrm{C}_{\mathrm{n}}$ (coloreado en azul para la columna de cada sistema Sn de la Tabla 2.4) al patrón de distancias de $S_{\mathrm{n}}$ que se repite, asi por ejemplo, las secuencias:

$\mathrm{C} 1 \in \in \mathrm{S} 1=2$

$\mathrm{C} 2 \in \in \mathrm{S} 2=2+4=6$

$\mathrm{C} 3 \in \in \mathrm{S} 3=4+2+4+2+4+6+2+6=30$

C $4 \in \in S 4=2+4+2+4+6+2+6+4+2+4+6+6+2+6+4+2+6+$ $4+6+8+4+2+4+2+4+8+6+4+6+2+4+6+2+6+6+4+2+4+$ $6+2+6+4+2+4+2+10+2+10=210$

$\mathrm{Y}$ así sucesivamente.

La razón de que en los sistemas hayan secuencias que se repiten, es porque en la determinación de las distancias de tal sistema, llegará el momento en que la primera frecuencia en las distancias del sistema coincidirá de nuevo en ubicación, como al inicio, con respecto a las frecuencias de las distancias de los sistemas anteriores, así por ejemplo, en la Tabla 2.1, en $\mathrm{P}_{3}=5$, la primera frecuencia que aparece está ubicada en el número 10 (por ser el primer factor de 5), que coincide en ubicación horizontal con una frecuencia de S1 también en 10 (por ser un factor de 2) y justo en la fila de arriba, en el número 9, aparece una frecuencia de S2 (por ser 9 un factor de 3) y estas frecuencias están entre los primos 7 y 11, cuya distancia es 4, que es la primera distancia en C3, como se muestra en la Tabla 2.5:

\section{TABLa 7}

Sección de la tabla de frecuencias de distribución entre los números primos 7 y 11

\begin{tabular}{|c|c|c|c|c|c|}
\hline 7 & - & - & - & - & \\
\hline 8 & $\mathrm{~F}_{1}$ & - & - & - & \\
\hline 9 & - & $\mathrm{F}_{2}$ & - & - & \\
\hline 10 & $\mathrm{~F}_{1}$ & - & $\mathrm{F}_{3}$ & - & \\
\hline 11 & - & - & - & - & - \\
\hline
\end{tabular}

Así:

$\mathrm{S}_{1}, \mathrm{P}_{1}=2, \mathrm{~F}_{1}$ está en 10

$\mathrm{S}_{2}, \mathrm{P}_{2}=3, \mathrm{~F}_{2}$ está en 9

$\mathrm{S}_{3}, \mathrm{P}_{3}=5, \mathrm{~F}_{3}$ está en 10

Ahora el lugar siguiente donde se vuelve a repetir esta misma ubicación de las frecuencias es:

\section{TABLA 8}

Sección de la tabla de frecuencias de distribución entre los números primos 37 y 41

\begin{tabular}{|c|c|c|c|c|c|}
\hline 37 & - & - & - & - & - \\
\hline 38 & $F_{1}$ & - & - & - & - \\
\hline 39 & - & $F_{2}$ & - & - & - \\
\hline 40 & $F_{1}$ & - & $F_{3}$ & - & - \\
\hline 41 & - & - & - & - & - \\
\hline
\end{tabular}

Así:

$\mathrm{S}_{1}, \mathrm{P}_{1}=2, \mathrm{~F}_{1}$ está en 40

$\mathrm{S}_{2}, \mathrm{P}_{2}=3, \mathrm{~F}_{2}$ está en 39

$\mathrm{S}_{3}, \mathrm{P}_{3}=5, \mathrm{~F}_{3}$ está en 40

Y estos valores están entre los números primos 37 y 41, cuya diferencia es 4 que es el primer valor de la segunda aparición de la secuencia $\mathrm{C}_{3}$.

Teorema 2.1. Las distancias $D_{\mathrm{k}} \in \in C_{\mathrm{n}}$ del sistema Sn construido hasta $P_{\mathrm{n}}$, se empiezan a sumar desde $P_{\mathrm{n}+1}$.

Demostración 2.5. Ya se sabe que en Sn el patrón de $C_{n}$ se vuelve a repetir, y las distancias $D k \in \in C_{n}$ del sistema $S_{n}$ se empiezan a sumar desde $P_{n+1}$, y no desde $\mathrm{P}_{\mathrm{n}}$, porque la distancia $\mathrm{D}_{0}=\mathrm{P}_{\mathrm{n}+1}-\mathrm{P}_{\mathrm{n}}$ pertenece al sistema $S_{n-1}$ ya que en adelante en $S n$ no se presentaran múltiplos de $\mathrm{P}_{\mathrm{n}}$ como primos, así cuando se repita $\mathrm{C}_{\mathrm{n}}$, el primer primo que inicie esta repetición que será igual a $\mathrm{P}_{\mathrm{k}}=\mathrm{x}\left(\mathrm{C}_{\mathrm{n}}\right)+\mathrm{P}_{\mathrm{n}+1}$, donde $\mathrm{x}$ va tomando valores tal que $\mathrm{x} \in \in \mathrm{N}$, no tendrá antes un múltiplo de $\mathrm{P}_{\mathrm{n}}$ a una distancia $\mathrm{D}_{0}$.

Teorema 2.2. El valor de la suma de las distancias de las secuencias de cada sistema coincide con el valor del producto de los primos con los que se construyó el sistema, es decir: 
$\sum_{k=1} D_{k}=C_{n}=P_{n} \# \sum_{k=1} D_{k}=C_{n}=P_{n}^{\#}$ Donde $D_{k} \in D_{k} \in C_{\mathrm{n}}$

Así por ejemplo, el sistema $\mathrm{S}_{3}$ se construyó con los números primos 2,3 y 5 , ahora el producto (primorial: $\left.P_{n} \# P_{n} \#\right) 2 * 3 * 5$ es $=30$, que coincide con el valor de la suma de las distancias de la secuencia $\mathrm{C}_{3}$, así $4+2+4+2+4+6+2+6=30$

Demostración. La razón por la cual la suma de las distancias de la secuencia de un sistema coincide con el producto de los primos con los que se construyó el sistema, es porque tal producto determina en qué lugar, o cada cuanto, se volverá a repetir la ubicación inicial del sistema con respecto a los sistemas anteriores, es decir, cuando la ubicación de las frecuencias del sistema del último primo encontrado se alinean en la misma ubicación inicial con las frecuencias de los sistemas anteriores hasta $S 1$, y esto se logra con el $m . c . m$, y como son primos, el $m$.c. $m=P_{n} \# P_{n} \#$, por ejemplo el $m$.c. $m$ de 2,3 y 5 es $2^{*} 3^{*} 5=30$ en $S_{3}$, y se representa lo anterior en la siguiente Tabla:

TABla 9

Construcción del m.c.m. de 2, 3 y 5

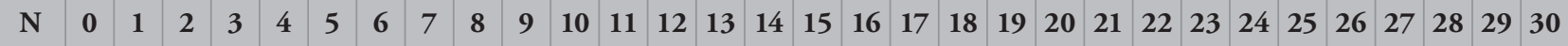

\begin{tabular}{|l|l|l|l|l|l|l|l|l|l|l|l|l|l|l|l|l|l|l|l|l|l|l|l|l|l|l|l|l|l|l|} 
F1 =2 & F1 & & F1 & & F1 & & F1 & & F1 & & F1 & & F1 & F1 & & F1 & & F1 & & F1 & & F1 & & F1 & & F1 & F1 & F1 \\
\hline F2 =3 & F2 & & F2 & & & F2 & & & F2 & & & F2 & & F2 & & & F2 & & & F2 & & & F2 & & & F2 & & F2 \\
\hline F3 =5 & F3 & & & & F3 & & & & & F3 & & & & & F3 & & & & & F3 & & & & & F3 & & & & F3 \\
\hline
\end{tabular}

Nótese que en la anterior tabla las frecuencias de 2,3 y 5 coinciden en alineación en cero y en 30 .

Así en la secuencia de $S_{2}=2-4$, sus distancias suman $2+4=6$, que coincide en el producto de $\mathrm{P}_{1}{ }^{*} \mathrm{P}_{2}=2^{*} 3=6$.

De esta manera se puede predecir que la suma de las distancias de la secuencia $\mathrm{C} 4$ de $\mathrm{S} 4$ es igual al producto $\mathrm{P}_{1} * \mathrm{P}_{2} * \mathrm{P}_{3} * \mathrm{P}_{4}=2 * 3 * 5 * 7=210$.

Teorema 2.3. $P_{\mathrm{k}}$ - DO es necesariamente un múltiplo de $P_{\mathrm{n}}$.

Demostración. $\mathrm{P}_{\mathrm{k}}=\mathrm{x}\left(\mathrm{C}_{\mathrm{n}}\right)+\mathrm{P}_{\mathrm{n}+1}$, por el Teorema 2.2. se sabe que $C_{n}=P_{n} \# C_{n}=P_{n} \#$, y $\mathrm{P}_{\mathrm{n}+1}=\mathrm{P}_{\mathrm{n}}+\mathrm{D}_{0}$, de manera que:

$\mathrm{P}_{\mathrm{k}}-\mathrm{D}_{0}=\mathrm{x}\left(\mathrm{C}_{\mathrm{n}}\right)+\mathrm{P}_{\mathrm{n}+1}-\mathrm{D}_{0}=\mathrm{x}\left(P_{n} \# P_{n} \#\right)+\mathrm{P}_{\mathrm{n}}$ $+\mathrm{D}_{0}-\mathrm{D}_{0}=\mathrm{x}\left(P_{n} \# P_{n} \#\right)+\mathrm{P}_{\mathrm{n}}, \mathrm{y}$ evidentemente $\mathrm{P}_{\mathrm{n}}$ $\mathrm{x}\left(P_{n} \# P_{n} \#\right)+\mathrm{P}_{\mathrm{n}}$

Teorema 2.4. El último número primo en cualquier repetición de $C_{\mathrm{n}}$ tiene la forma $x\left(P_{\mathrm{n}} \#\right)+P_{\mathrm{n}}+1$.
Demostración. Por el Teorema 2.1. se sabe que las distancias $\mathrm{D}_{\mathrm{k}} \in \in \mathrm{C}_{\mathrm{n}}$ del sistema $\mathrm{Sn}$ construido hasta $\mathrm{P}_{\mathrm{n}}$, se empiezan a sumar desde $\mathrm{P}_{\mathrm{n}+1}$, es decir que los números primos determinados en $\mathrm{Sn}$ son el resultado de ir sumando a $\mathrm{P}_{\mathrm{n}+1}$ cada distancia, pero la suma de estas distancias genera un patrón que se repite llamado $\mathrm{xC}_{\mathrm{n}}$, donde $\mathrm{x}$ es el número de su repetición, según la Demostración 2.4, así que el valor de la suma de las distancias hasta completas la secuencia más $\mathrm{P}_{\mathrm{n}+1}$ es un número primo determinado por $\mathrm{Sn}, \mathrm{y}$ como, según el Teorema 2.2. , $\sum_{k=1} D_{k}=C_{n}=P_{n} \#$ $\sum_{k=1} D_{k}=C_{n}=P_{n} \#$ Donde $D_{k} \in D_{k} \in \mathrm{C}_{\mathrm{n}}$ y $P_{n} \#$ $P_{n} \#$ es el primorial hasta $P_{n} P_{n}$, entonces el ultimo numero último número primo en cualquier repetición de $\mathrm{C}_{\mathrm{n}}$ tiene la forma $\mathrm{x}\left(\mathrm{P}_{\mathrm{n}} \#\right)+\mathrm{P}_{\mathrm{n}+1}$, donde $\mathrm{x}$ va tomando valores tal que $\mathrm{x} \in \in \mathrm{N}$.

Teorema 2.5. El número 1 no es un número primo.

Demostración. En el estudio anterior se definieron los sistemas de distribución desde $S_{1}$, ahora considérese la existencia de $S_{0}$. 
Recuérdese que los sistemas de distribución se construyen a partir de las frecuencias y distancias de distribución, considérese el sistema $S_{1}$ construido por el numero primo $\mathrm{P}_{1}=2$, en este sistema la frecuencia $\mathrm{F}_{1}$ son los múltiplos de $\mathrm{P}_{1}$, es de decir, la multiplicación de 2 por todos los números naturales $\mathrm{N}>1$, ya que si se multiplica por 1, el resultado sería que el mismo $P_{1}$ sería una frecuencia, lo que implicaría que $\mathrm{P}_{1}$ no es primo ya que $\mathrm{F} \neq \mathrm{P}$, lo que lleva a una contradicción, ya que $\mathrm{P}_{1} \in \mathrm{P}$.

Ahora suponer la existencia de un sistema $S_{0}$, supone la existencia de un numero primo anterior a 2 , y el único número natural diferente de cero anterior a 2 es 1 , así $\mathrm{P}_{0}=1$. Ahora para construir el $\mathrm{S}_{0}$ se determinan sus frecuencias que serán los múltiplos de $\mathrm{P}_{0}=1$, es decir el producto de $\mathrm{P}_{0}=1$ con todos los números naturales mayores a 1 , pero se sabe que todo número multiplicado por 1 da él mismo, lo que significa que las frecuencias $\mathrm{F}_{0}$ son todos los números naturales mayores a $1, y$ como $\mathrm{F}_{0} \neq \mathrm{P}$, esto quiere decir que, según $\mathrm{S}_{0}$, no existirán más números primos a excepción de $\mathrm{P}_{0}=1$, lo cual no es cierto, por ello el $\mathrm{S}_{0}$ es inexistente, y $\mathrm{P}_{0}$ no existe, de ahí se concluye que 1 no es primo.

Teorema 2.6. Para $S_{n}, x\left(P_{n} \#\right)+1$ será un numero primo, y será el penúltimo número primo determinado por $C_{\mathrm{n}}$.

Demostración. Como en la Demostración del Teorema 2.1., llámese:

$D_{0}=P_{n+1}-P_{n}, D_{-1}=P_{n+1}-P_{n-1}, D_{-2}=P_{n+1}-P_{n-2}, D_{-3}=P_{n+1}$ $-P_{n-3}, \ldots \ldots \ldots, D_{-j}=P_{n+1}-P_{n-j}$

Donde $\mathrm{P}_{\mathrm{n}-\mathrm{i}}=\mathrm{P}_{1}=2$

Así que $\mathrm{P}_{\mathrm{n}+1}=\mathrm{P}_{\mathrm{n}-\mathrm{j}}+\mathrm{D}_{-\mathrm{j}}$

Recuérdese que $\mathrm{P}_{\mathrm{k}}=\mathrm{x}\left(\mathrm{C}_{\mathrm{n}}\right)+\mathrm{P}_{\mathrm{n}+1} \mathrm{y}$ :

$\mathrm{P}_{\mathrm{k}}-\mathrm{D}_{-\mathrm{j}}=\mathrm{x}\left(\mathrm{C}_{\mathrm{n}}\right)+\mathrm{P}_{\mathrm{n}+1}-\mathrm{D}_{-\mathrm{j}}=\mathrm{x}\left(P_{n} \#\right)+\mathrm{P}_{\mathrm{n}-\mathrm{j}}+\mathrm{D}_{-\mathrm{j}}-\mathrm{D}_{-\mathrm{j}}=$ $\mathrm{x}\left(P_{n} \#\right)+\mathrm{P}_{\mathrm{n}-\mathrm{j}}$, y como $\mathrm{P}_{\mathrm{n}-\mathrm{j}}<\mathrm{P}_{\mathrm{n}}$ evidentemente

$P_{n-j} \mid x\left(P_{n} \#\right)+P_{n-j}$

Así que $\mathrm{P}_{\mathrm{k}}-\mathrm{D}_{-\mathrm{j}}$ no es un numero primo, así que el único número anterior a $\mathrm{P}_{\mathrm{n}-\mathrm{j}}=\mathrm{P}_{1}=2$ es 1 , es decir que se debe retrocedes una unidad ya que $2-1=1$, pero por el Teorema 2.5., 1 no es un numero primo, de manera que $\mathrm{x}\left(P_{n} \#\right)+\mathrm{P}_{\mathrm{n}-\mathrm{j}}-1=\mathrm{x}\left(P_{n} \#\right)+2-1=\mathrm{x}\left(P_{n} \#\right)+1$ no es un múltiplo de ningún numero primo, al menos hasta $\mathrm{P}_{\mathrm{n}}$, de manera que para $\mathrm{S}_{\mathrm{n}}, \mathrm{x}\left(P_{n} \#\right)+1$ será un numero primo, y será el penúltimo número primo determinado por $\mathrm{C}_{\mathrm{n}}$.

Teorema 2.7. La ultima distancia $D_{\mathrm{k}} \in C_{\mathrm{n}}$ es igual a $P_{\mathrm{n}+1}-1$.

Demostración. Ahora como el último número primo en cualquier repetición de $\mathrm{C}_{\mathrm{n}}$ tiene la forma $x\left(P_{n} \#\right)+P_{n+1}$ según el Teorema 2.4., y el penúltimo número primo determinado por $\mathrm{C}_{\mathrm{n}}$ tiene la forma $\mathrm{x}\left(P_{n} \#\right.$ ) + 1 según el Teorema 2.6., la diferencia del ultimo primo y el penúltimo primo en $\mathrm{C}_{\mathrm{n}}$ es:

$\mathrm{x}\left(\mathrm{P}_{\mathrm{n}} \#\right)+\mathrm{P}_{\mathrm{n}+1}-\left(\mathrm{x}\left(P_{n} \#\right)+1\right)=\mathrm{P}_{\mathrm{n}+1}-1$ que es la última distancia $\mathrm{D}_{\mathrm{k}} \in \mathrm{C}_{\mathrm{n}}$

Teorema 2.8. Cada sistema $S_{\mathrm{n}}$ funcionará perfectamente en la determinación de números primos hasta llegar al cuadrado del primo $P_{\mathrm{n}+1}$, siguiente al primo $P_{\mathrm{n}}$ hasta donde se construyó el sistema.

Demostración: El cuadrado del primo siguiente al primo hasta donde se construyó el sistema, es el primer número que no es múltiplo de los primos con los que se construyó el sistema, por ello no se puede considerar como frecuencia. Esto se explica Así:

$$
\begin{aligned}
& F_{1}=P_{1} * N, F_{2}=P_{2} * N, F_{3}=P_{3} * N \\
& \ldots \ldots \ldots \ldots \ldots, F_{n}=P_{n} * N
\end{aligned}
$$

Donde $\mathrm{N}$ son los números naturales mayores a 1 .

$\left(F_{1}\right) \in S_{1},\left(F_{1}, F_{2}\right) \in S_{2},\left(F_{1}, F_{2}, F_{3}\right) \in S_{3}, \ldots \ldots$, $\left(F_{1}, F_{2}, F_{3}, \ldots \ldots, F_{n}\right) \in S_{n}$

Ahora en $S_{n \text { considerese }}$ $\left(P_{n+1}\right)^{2}=\left(P_{n+1}\right)\left(P_{n+1}\right)=\left(P_{n+1}\right) * N=F_{n+1}$

Pero $F_{n+1} \notin S_{n}$ 
De manera que la aparición de $\left(P_{n+1}\right)^{2}=F_{n+1}$ en $S_{n}$ hace que $S_{n}$ sea correcta en la determinación de números primos hasta llegar a $\left(P_{n+1}\right)^{2}$, de ahí en adelante el sistema $S_{n}$ determinará tanto números primos como números primos falsos que serán todo tipo de combinación de productos de números primos diferentes a los números primos de las frecuencias pertenecientes a $S_{n}$. así por ejemplo, el $S_{1}$ se construyó con el primo 2, y el siguiente número primo de 2 es 3 , y el cuadrado de 3 es 9, así $S_{1}$ funcionara bien hasta llegar a 9, ya que 9 no es primo, y a partir de ahí el $S_{1}$ predecirá tanto números primos como números impares compuestos; ahora $S_{2}$ se construyó con los primos 2 y 3 , y el siguiente primo de 3 es 5 , y el cuadrado de 5 es 25 , es decir, que $\mathrm{S}_{2}$ funcionara perfectamente hasta llegar a 25 , ya que 25 no es primo.

Demostración 2.5. Llámese defecto de secuencia al hecho de que en un sistema $S_{\mathrm{n}}$ se supere el valor de $P_{n+1}^{2}$ antes de terminar la primera secuencia, de manera que $P_{n+1}+C_{n}=P_{n+1}+P_{n} \#>P_{n+1}^{2}$.

Demostración 2.6. Llámese producto de fusión, a aquel producto que en la construcción de un sistema hace que se fusionen o unan dos distancias. Los productos de fusión en $S_{\mathrm{n}}$ son los productos de $P_{\mathrm{n}}$ con los primos determinados con las distancias de la secuencia $C_{\mathrm{n}-1}$, es decir, con los números que son diferentes a los primos anteriores a $P_{\mathrm{n}}$ o a sus múltiplos, esto para que en $S_{\mathrm{n}}$ los múltiplos de $P_{\mathrm{n}}$ ya no sean considerados como primos Asi por ejemplo en la TABLA 2.8, se observa que en $S_{2}$ hay una distancia 4, resultado de la fusión de las dos distancias 2 y 2 de $S_{1}$, presentes entre los números primos 7 y 11. Asi mismo se llamara distancias de fusión a las distancias resultado de fusionar dos distancias del sistema anterior por un producto de fusión.

\section{Tabla 10}

Ejemplo de producto de fusión, tomado del cuadro de las distancias de los sistemas de distribución

\begin{tabular}{|c|c|c|}
\hline $\mathbf{N}$ & $\mathbf{S}_{1}$ & $\mathbf{S}_{2}$ \\
\hline $\mathbf{3}$ & 2 & \\
\hline $\mathbf{5}$ & 2 & \\
\hline $\mathbf{5}$ & 2 & 2 \\
\hline $\mathbf{7}$ & 2 & \\
\hline $\mathbf{7 n}$ & 2 & \\
\hline & \multirow{2}{*}{2} & \\
\hline $\mathbf{1 1}$ & 2 & \\
\hline
\end{tabular}

Así un producto de fusión en $S_{n}$ elimina un falso número primo construido por $\mathrm{S}_{\mathrm{n}-1}$, $\mathrm{y}$ lo define como múltiplo de $\mathrm{P}_{\mathrm{n}}$.

Demostración 2.7. Llámese bloque de secuencia $\boldsymbol{b}_{\mathrm{k}}$ a la suma de las distancias de una secuencia de un sistema desde $D_{1}$ o desde la distancia siguiente a la distancia en que termina el bloque anterior, hasta aquella distancia siguiente que es una fusión de dos distancias del sistema inmediatamente anterior, asi por ejemplo, en el cuadro de las distancias de los sistemas de distribución, los dos bloques del sistema $S_{3}$ en su secuencia son:

\section{TABla 11}

Bloques de $\mathrm{S}_{2}$, tomado del cuadro de las distancias de los sistemas de distribución

\begin{tabular}{|c|c|c|c|c|}
\hline $\mathbf{N}$ & $S_{1}$ & $S_{2}$ & $S_{3}$ & \\
\hline 3 & \multirow{2}{*}{2} & & & \\
\hline 5 & & & & \\
\hline 5 & \multirow{2}{*}{2} & \multirow{2}{*}{2} & & \\
\hline 7 & & & & \\
\hline 7 & 2 & \multirow{3}{*}{4} & \multirow{3}{*}{4} & \multirow{5}{*}{$\begin{array}{c}\text { BLOQUE } 1 \\
\text { DEL } \\
\text { SISTEMA } 3\end{array}$} \\
\hline & & & & \\
\hline 11 & 2 & & & \\
\hline 11 & \multirow{2}{*}{2} & \multirow{2}{*}{2} & \multirow{2}{*}{2} & \\
\hline 13 & & & & \\
\hline
\end{tabular}




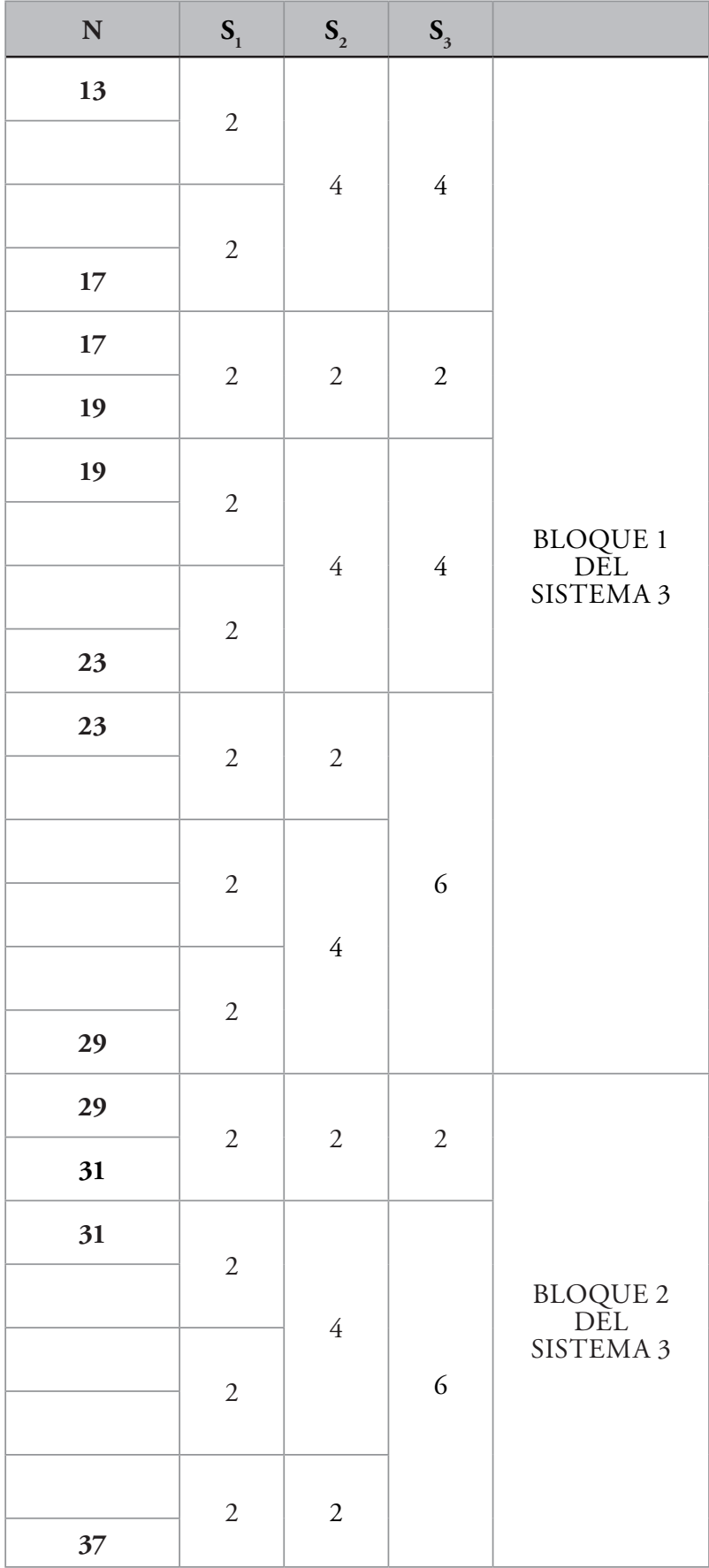

Demostración 2.8. Llámese productos de fusión de defecto de secuencia de $S_{\mathrm{n}}$ a los productos de fusión de todos los sistemas $S_{\mathrm{n}+\mathrm{k}^{\mathrm{k}}}$ con $k \geq 1$, esto es, todo tipo infinitos de combinación y repetición de productos de primos de la forma $P_{\mathrm{n}+\mathrm{k}}$ con $k \geq 1$, estos productos no son primos, pero para $S_{\mathrm{n}}$ si lo son, por eso están en el defecto de secuencia.
Teorema 2.9. Sea $R_{\mathrm{n}}$ la cantidad de productos de fusión que unen distancias en un sistema $S_{n}$ y sea $Q_{n-1}$ la cantidad de distancias existentes en un $C_{\mathrm{n}-1}$, de manera que $R_{\mathrm{n}}=Q_{\mathrm{n}-1}$.

Demostración. La cantidad de productos de fusión $R_{n}$ presentes en un $S_{n}$ es igual a los productos de $\mathrm{P}_{\mathrm{n}}$ con los primos determinados con las distancias de la secuencia $\mathrm{C}_{\mathrm{n}-1}$, esto para que en $\mathrm{S}_{\mathrm{n}}$ los múltiplos de $P_{n}$ ya no sean considerados como primos, sin incluir a $\mathrm{P}_{\mathrm{n}-1}$, ni a los primos anteriores, ya que los múltiplos de estos mismos ya se excluyeron de ser números primos en los sistemas anteriores. Sea $\mathrm{Q}_{\mathrm{n}-1}$ la cantidad de distancias existentes en un $\mathrm{C}_{\mathrm{n}-1}$, que es igual a la cantidad de primos determinados con las distancias de la secuencia $C_{n-1}$, de manera que $R_{n}=Q_{n-1}$.

Teorema 2.10. El primer producto de fusión en $C_{\mathrm{n}}$ es $P_{n}^{2}$, y el segundo producto de fusión es $P_{n} P_{n+1}$.

Demostración. Como se explicó en la Demostración del Teorema 2.9., los productos de fusión en $\mathrm{C}_{\mathrm{n}}$ es igual a los productos de $\mathrm{P}_{\mathrm{n}}$ con los primos determinados con las distancias de la secuencia $\mathrm{C}_{\mathrm{n}-1}$, esto para que en $\mathrm{S}_{\mathrm{n}}$ los múltiplos de $\mathrm{P}_{\mathrm{n}}$ ya no sean considerados como primos, sin incluir a $\mathrm{P}_{\mathrm{n}-1}$, ni a los primos anteriores, pero si a $\mathrm{P}_{\mathrm{n}}$ y a los primos posteriores determinados con las distancias de la secuencia $\mathrm{C}_{\mathrm{n}-1}$, ya que los múltiplos de $\mathrm{P}_{\mathrm{n}-1}$, y de los primos anteriores ya se excluyeron de ser números primos en los sistemas anteriores, pero los múltiplo de $\mathrm{P}_{\mathrm{n}} \mathrm{y}$ de los primos posteriores aún no se han excluido. Los múltiplos de $\mathrm{P}_{\mathrm{n}}$ son de la forma $\mathrm{P}_{n}{ }^{*} \mathrm{~N}$, pero para que $\mathrm{P}_{n}{ }^{*} \mathrm{~N}$ sea un producto de fusión en $\mathrm{C}_{\mathrm{n}}, \mathrm{N} \geq \mathrm{P}_{\mathrm{n}}$, ya que si $\mathrm{N}<\mathrm{P}_{\mathrm{n}}$, $\mathrm{N}$ tomara valores de primos anteriores a $\mathrm{P}_{n}$ o números anteriores a $\mathrm{P}_{n}$ compuestos por primos anteriores a $\mathrm{P}_{n}$. así que $\mathrm{P}_{n}{ }^{*} \mathrm{~N}$ con $\mathrm{N}$ $=\mathrm{P}_{\mathrm{n}}$, es un producto de fusión en $\mathrm{C}_{\mathrm{n}}$.

Considérese los dos casos diferentes:

- El primer producto de fusión en $\mathrm{C}_{n}$ es $\mathrm{P}_{n} * \mathrm{P}_{n-\mathrm{k}}, \mathrm{P}_{\mathrm{n}-\mathrm{k}}<$ $\mathrm{P}_{\mathrm{n}}$, así que $\mathrm{P}_{\mathrm{n}-\mathrm{k}}$ es anterior a $\mathrm{P}_{\mathrm{n}}$, lo que quiere decir que ya habrá creado antes de $S_{n}$ un sistema $S_{n-k}$ en el que se habrán excluido los múltiplos de $\mathrm{P}_{\mathrm{n}-\mathrm{k}}, \mathrm{y}_{\mathrm{n}}$ * 
$\mathrm{P}_{\mathrm{n}-\mathrm{k}}$ sería un múltiplo de $\mathrm{P}_{\mathrm{n}-\mathrm{k}}$, de manera que en $\mathrm{S}_{\mathrm{n}}$ no hay necesidad de eliminar el numero primo falso $\mathrm{P}_{\mathrm{n}} * \mathrm{P}_{\mathrm{n}-\mathrm{k}}$, porque ya ha sido eliminado por el sistema $S_{n-k}$, por ello el primer producto de fusión en $C_{n}$ no es $\mathrm{P}_{\mathrm{n}}{ }^{*} \mathrm{P}_{\mathrm{n}-\mathrm{k}}$.

- El primer producto de fusión en $\mathrm{C}_{n}$ es $\mathrm{P}_{n}{ }^{*} \mathrm{P}_{n+k}$, donde $P_{n+k}$ fue determinado en $C_{n-1}, P_{n+k}>P_{n}$, así que $P_{n+k}$ será posterior a $\mathrm{P}_{\mathrm{n}}$, así que $\mathrm{P}_{\mathrm{n}}{ }^{*} \mathrm{P}_{\mathrm{n}+\mathrm{k}}$ si es un producto de fusión en $\mathrm{C}_{\mathrm{n}}$ pero no será el primero, ya que $\mathrm{P}_{\mathrm{n}}$ * $\mathrm{P}_{\mathrm{n}}$ también es un producto de fusión en $\mathrm{C}_{\mathrm{n}}, \mathrm{y}_{\mathrm{n}}{ }^{*} \mathrm{P}_{\mathrm{n}}$ $<\mathrm{P}_{n} * \mathrm{P}_{n+k}$, por eso el primer producto de fusión en $\mathrm{C}_{\mathrm{n}}$ no es $\mathrm{P}_{\mathrm{n}} * \mathrm{P}_{\mathrm{n}+\mathrm{k}}$.

Así queda demostrado que el primer producto de fusión en $\mathrm{C}_{\mathrm{n}}$ es $\mathrm{P}_{\mathrm{n}}{ }^{*} \mathrm{P}_{\mathrm{n}}=P_{n}^{2}$.

Bien, $P_{n} P_{n+k}>P_{n} P_{n+1}$ para $\mathrm{k}>1$, de manera que ninguno de los productos de fusión de la forma $P_{n} P_{n+k}$ para k>1 es el segundo producto de fusión.

Solo resta demostrar que $P_{n}^{3}>P_{n} P_{n+1}$, ahora Considérese el importante postulado de Bertrand: "para todo entero positivo $\mathrm{n}$ existe un primo $\mathrm{P}$ tal que $\mathrm{n}<\mathrm{P} \leq 2 \mathrm{n}$ " (Niven y Zukcerman 1969, 181).

Sea $\mathrm{n}=\mathrm{P}_{\mathrm{n}}$, así se tiene que $\mathrm{P}_{\mathrm{n}}<\mathrm{P}_{\mathrm{n}+1}<2 \mathrm{P}_{\mathrm{n}}$, supóngase que $\mathrm{P}_{n+1}=2 \mathrm{P}_{n}$, de manera que $P_{n} P_{n+1}=P_{n} 2 P_{n}=2 P_{n}^{2}$ , y $2 P_{n}^{2}<P_{n}^{3}$, para $P_{n}>2$, pero con comprobación numérica se ve que el segundo producto de fusión es $P_{n} P_{n+1}$ para $\mathrm{P}_{\mathrm{n}} \geq 2$.

Teorema 2.11. El último producto de fusión en $C_{\mathrm{n}}$ es igual a $P_{\mathrm{n}}$ por el penúltimo número primo determinado por $C_{\mathrm{n}-1}, y$ es de la forma $x\left(P_{n} \#\right)+P_{n}$.

Demostración. Por la Demostración del Teorema 2.6., se sabe que el penúltimo número primo determinado por $\mathrm{C}_{\mathrm{n}-1}$ es de la forma $\mathrm{x}\left(\mathrm{P}_{\mathrm{n}-1} \#\right)+1$, y por la Demostración del Teorema 2.3., se sabe que $\mathrm{x}\left(\mathrm{P}_{\mathrm{n}} \#\right)+\mathrm{P}_{\mathrm{n}}$ es el producto de fusión que hace que la última distancia $\mathrm{D}_{\mathrm{k}} \in \mathrm{C}_{\mathrm{n}}$ sea una fusión, ahora haciendo la división: $\frac{\mathrm{x}\left(P_{n} \#\right)+P_{n}}{P_{n}}=\mathrm{x}\left(P_{n-1} \#\right)+1$ que es el penúltimo numero primo determinado por $\mathrm{C}_{\mathrm{n}-1}$.

Teorema 2.12. El penúltimo producto de fusión en $C_{\mathrm{n}}$ es igual a $P_{\mathrm{n}} \#-P_{\mathrm{n}}$.

Demostración. Por el Teorema 2.11 se sabe que el último producto de fusión en $\mathrm{C}_{\mathrm{n}}$ es igual a $\mathrm{P}_{\mathrm{n}}$ por el penúltimo número primo determinado por $\mathrm{C}_{\mathrm{n}-1}$, así que el penúltimo producto de fusión en $\mathrm{C}_{\mathrm{n}}$ es igual a $\mathrm{P}_{\mathrm{n}}$ por el antepenúltimo número primo determinado por $\mathrm{C}_{\mathrm{n}-1}$, $\mathrm{Y}$ el antepenúltimo número primo determinado por $\mathrm{C}_{\mathrm{n}-1}$ es de la forma $\mathrm{x}\left(P_{n-1} \#\right)-1$, ya que $P_{n-1}$ no divide a $\mathrm{x}\left(P_{n-1} \#\right)-1$, de manera que $P_{n}\left(P_{n-1} \#-1\right)=P_{n} \#-P_{n}$.

Teorema 2.13. Entre el último producto de fusión y el penúltimo existen dos números considerados primos por $S_{n}$.

Demostración. Sea el último producto de fusión en $\mathrm{C}_{\mathrm{n}}$ de la forma $\left(P_{n} \#\right)+P_{n}$ o $\left(P_{n} \#\right)+\mathrm{K}$, y sea el penúltimo producto de fusión en $\mathrm{C}_{\mathrm{n}}$ de la forma $\mathrm{P}_{\mathrm{n}}$ \#$\mathrm{P}_{\mathrm{n}}$ o $\mathrm{P}_{\mathrm{n}} \#$ - $\mathrm{K}$, donde $\mathrm{K}=P_{n}$, ahora si a $\mathrm{K}$ se le dan valores enteros menores a $P_{n}$, las expresiones $\left(P_{n} \#\right)+\mathrm{K}$ y $\mathrm{P}_{\mathrm{n}}$ \#- K serán divisibles por los números primos anteriores a $P_{n}$, exceptuando a $K=1$, ya que las dos expresiones $\left(P_{n} \#\right)+1$ y $\mathrm{P}_{\mathrm{n}} \#-1$ son las únicas que no son divisibles por los números primos menores o igual a $\mathrm{P}_{\mathrm{n}}$, de manera que para $S_{\mathrm{n}},\left(P_{n} \#\right)+1_{\text {y }} \mathrm{P}_{\mathrm{n}} \#-1$ son primos.

Teorema 2.14. La cantidad de distancias $Q_{\mathrm{n}}$ existentes en un $C_{\mathrm{n}}$, es igual al producto de la cantidad de distancias $Q_{\mathrm{n}-1}$ existentes en un $C_{\mathrm{n}-1}$ por el número primo $P_{\mathrm{n}}$, menos la cantidad de distancias $Q_{\mathrm{n}-1}$. asi:

$$
Q_{n}=Q_{n-1} P_{n}-Q_{n-1}=Q_{n-1}\left(P_{n}-1\right)
$$

Demostración. Supóngase que se conoce la cantidad de distancias $\mathrm{Q}_{\mathrm{n}}$ existentes en un $\mathrm{C}_{\mathrm{n}}$, y se desea saber cuánta cantidad de distancias $\mathrm{Q}_{n+1}$ existentes en un $\mathrm{C}_{n+1}$, para ello se calcula cuantas veces esta $\mathrm{C}_{n}$ en $\mathrm{C}_{n+1}$, y como por el Teorema 2.2. $\mathrm{C}_{n}=\mathrm{P}_{n} \#$ y $\mathrm{C}_{\mathrm{n}+1}=\mathrm{P}_{\mathrm{n}+1} \#$, de manera que: 


$$
\frac{C_{n+1}}{C_{n}}=\frac{P_{n+1} \#}{P_{n} \#}=P_{n+1}
$$

De manera que $C_{n}$ está $P_{n+1}$ veces en $C_{n+1}$, que sería lo mismo decir $Q_{n}$ esta $P_{n+1}$ veces en $Q_{n+1}$, sin embargo esta cantidad de distancias merma por los productos de fusión que construyen a $S_{n+1}$, y por el Teorema 2.9. $\mathrm{R}_{\mathrm{n}+1}=\mathrm{Q}_{\mathrm{n}}$, por esto:

$$
Q_{n+1}=Q_{n} P_{n+1}-Q_{n}=Q_{n}\left(P_{n+1}-1\right)
$$

Ahora $\mathrm{Q}_{1}=1$, y este es el único $Q_{n}$ que no se define por la formula $Q_{n}=Q_{n-1}\left(P_{n}-1\right)$, ya que $\mathrm{Q}_{0}$ no existe. Así:

$$
\begin{aligned}
& Q_{2}=Q_{1}\left(P_{2}-1\right)=2 \\
& Q_{3}=Q_{2}\left(P_{3}-1\right)=8 \\
& Q_{4}=Q_{3}\left(P_{4}-1\right)=48 \\
& Q_{5}=Q_{4}\left(P_{5}-1\right)=480 \\
& Q_{6}=Q_{5}\left(P_{6}-1\right)=5.760 \\
& Q_{7}=Q_{6}\left(P_{7}-1\right)=92.160 \\
& Q_{8}=Q_{7}\left(P_{8}-1\right)=1.658 .880 \\
& Q_{9}=Q_{8}\left(P_{9}-1\right)=36.495 .360 \\
& Q_{10}=Q_{9}\left(P_{10}-1\right)=1.021 .870 .080 \\
& Y \text { así sucesivamente. }
\end{aligned}
$$

Teorema 2.15. $Q_{n+1}$ aumenta su proporción con respecto a $Q_{n}$ al crecer el valor de $n$, asi $\lim _{n \rightarrow \infty} \frac{Q_{n}}{Q_{n+1}}=0$. Demostración. $Q_{n}=Q_{n-1}\left(P_{n}-1\right)$, así $Q_{n+1}=Q_{n}\left(P_{n+1}-1\right)=Q_{n-1}\left(P_{n}-1\right)\left(P_{n+1}-1\right)$, ahora $\frac{Q_{n}}{Q_{n+1}}=\frac{Q_{n-1}\left(P_{n}-1\right)}{Q_{n-1}\left(P_{n}-1\right)\left(P_{n+1}-1\right)}=$ 1

$\overline{\left(P_{n+1}-1\right)}$,

y evidentemente $\lim _{n \rightarrow \infty} \frac{1}{\left(P_{n+1}-1\right)}=0$.

Teorema 2.16. $Q_{\mathrm{n}}$ aumenta su proporción con respecto a $R_{\mathrm{n}}$, asi $\lim _{n \rightarrow \infty} \frac{\left(R_{n}\right)}{\left(Q_{n}\right)}=0$, de manera que $R_{\mathrm{n}}$ se ha repartido en $C_{\mathrm{n}}$ con un $Q_{\mathrm{n}}$ cada vez más mayor con respecto a $R_{\mathrm{n}}$.

Demostración. Por el Teorema 2.9 se sabe que
$R_{n}=Q_{n-1}$, y por el Teorema 2.14. se

tiene que $Q_{n}=Q_{n-1}\left(P_{n}-1\right)$, ahora

$\frac{Q_{n-1}}{Q_{n-1}\left(P_{n}-1\right)}=\frac{1}{\left(P_{n}-1\right)}, \lim _{n \rightarrow \infty} \frac{1}{\left(P_{n+1}-1\right)}=0$

Teorema 2.17. $P_{n}^{2}>P_{n+1}+D_{1}$, para $n>1$.

Demostración. Considérese el postulado de Bertrand: $\mathrm{n}<\mathrm{P} \leq 2 \mathrm{n}$

Sea $n=P_{n}$ así $P_{n}<P_{k} \leq 2 P_{n}$, pero si $P_{n}$ y $P_{k}$ son primos, entonces $\mathrm{P}_{k}<2 \mathrm{P}_{n}$, ahora puede considerarse que $\mathrm{P}_{\mathrm{k}}=\mathrm{P}_{\mathrm{n}+1}$.

Ahora $\left(2 \mathrm{P}_{\mathrm{n}}\right)<P_{k_{l}}<2\left(2 \mathrm{P}_{\mathrm{n}}\right)=4 \mathrm{P}_{\mathrm{n}}$, y por el Teorema 2.10. se sabe que $P_{n}^{2}$ es el primer producto de fusión, y $4 \mathrm{P}_{\mathrm{n}}<P_{n}^{2}$ si $4<\mathrm{P}_{\mathrm{n}}$, y $\mathrm{P}_{\mathrm{n}}>4$ para $\mathrm{n}>2$. Así si $4 \mathrm{P}_{\mathrm{n}}<$ $P_{n}^{2}$ entonces $P_{k_{l}}<P_{n}^{2}$, y como $P_{k_{l}}>\mathrm{P}_{\mathrm{k}}$, entonces puede ser $P_{k_{l}}=P_{n+1}+D_{1}$, lo que demuestra que $P_{n}^{2}>P_{n+1}+D_{1}$, para $\mathrm{n}>2$, pero con comprobación se ve que $P_{n}^{2}>P_{n+1}+D_{1}$, para $\mathrm{n}>1$.

Teorema 2.18. La primera distancia $D_{1} \in C_{\mathrm{n}}$ para $n>1$, no es una fusión, de manera que $D_{1} \in C_{\mathrm{n}}$ es la segunda distancia $D_{2} \in C_{\mathrm{n}-1}$.

Demostración. Por el Teorema 2.17 se sabe que $P_{n}^{2}>P_{n+1}+D_{1}$ para $\mathrm{n}>1$, y se sabe por el Teorema 2.10 que el primer producto de fusión en $\mathrm{C}_{\mathrm{n}}$ es $P_{n}^{2}$, de manera que al ser $P_{n}^{2}>P_{n+1}+D_{1}, P_{n}^{2}$ no puede estar dentro de $D_{1}$ haciéndola una fusión.

Teorema 2.19. La última distancia $D_{\mathrm{k}} \in C_{\mathrm{n}}$ es una fusión.

Demostración. Como dice el Teorema 2.7 la última distancia $D_{k} \in C_{n}$ es igual a $P_{n+1}-1$, que es una fusión, y como se vio en la Demostración del mismo Teorema, se tiene que:

$$
\begin{aligned}
& \mathrm{P}_{\mathrm{n}}\left|\mathrm{x}\left(P_{n} \#\right)+\mathrm{P}_{\mathrm{n}}, \mathrm{P}_{\mathrm{n}-1}\right| \mathrm{x}\left(P_{n} \#\right)+\mathrm{P}_{\mathrm{n}-1}, \mathrm{P}_{\mathrm{n}-2} \mid \mathrm{x}\left(P_{n} \#\right)+ \\
& \mathrm{P}_{\mathrm{n}-2}, \ldots \ldots . ., \mathrm{P}_{\mathrm{n}-\mathrm{j}} \mid \mathrm{x}\left(P_{n} \#\right)+\mathrm{P}_{\mathrm{n}-\mathrm{j}},
\end{aligned}
$$


Así que los múltiplos de los primos anteriores a $\mathrm{P}_{\mathrm{n}}$ ya han sido descartados como primos por sus sistemas correspondientes, pero se sabe que $\mathrm{x}\left(P_{n} \#\right)+\mathrm{P}_{\mathrm{n}}$ no es primo por ser múltiplo de $\mathrm{P}_{\mathrm{n}}$, solamente desde $S_{\mathrm{n}}$, ya que en los sistemas anteriores hasta $S_{n-1}, x\left(P_{n} \#\right)+P_{n}$ si se consideraba primo, y recuérdese por la Demostración 2.6 que productos de fusión es el producto de $\mathrm{P}_{\mathrm{n}}$ por los números diferentes a los números primos anteriores a $\mathrm{P}_{\mathrm{n}}$ o a sus múltiplos, así cualquier $\mathrm{P}_{\mathrm{n}-\mathrm{k}} \mid \mathrm{x}($ $\left.P_{n} \#\right)+\mathrm{P}_{\mathrm{n}}$, por ello $\mathrm{x}\left(P_{n} \#\right)+\mathrm{P}_{\mathrm{n}}$ es un producto de fusión que une dos distancias de $\mathrm{C}_{\mathrm{n}-1} \mathrm{y}$ crea la última distancia $P_{n+1}-1$ en $C_{n}$.

Demostración 2.9. Llámese simetría secuencial $x_{\text {sim }}$, al hecho de que en las distintas repeticiones de $P_{n} \#$ se presenta simetría en los numeros primos que incluye $S_{n}$, sin tomar en cuenta los números primos anteriores a $P_{n+1}$, presentes en las dos secciones resultado de dividir $P_{n} \#$ en 2, donde $x \in N$ representa el numero ordinal de la simetría, asi por ejemplo 1 sim $_{\mathrm{n}}$ representa la simetría en las dos secciones que están entre 0 y $P_{n} \#$, $2 \operatorname{sim}_{\mathrm{r}}$ representa la simetría en las dos secciones que están entre $P_{n} \#$, y $2 P_{n} \#, 3$ sim representa la simetría en las dos secciones que están entre $2 P_{n}^{\#}, y 3 P_{n} \#$, y xsim representa la simetría en las dos secciones que están entre $(x-1) P_{n} \#, y x P_{n} \#$.

\section{TABLA 12}

Ejemplo de simetría en 1 sim, tomado de la tabla de frecuencias de distribución

\begin{tabular}{|c|c|}
\hline $\mathbf{N}$ & $\mathbf{P}_{3}$ \\
\hline 1 & \\
\hline 2 & \\
\hline 3 & \\
\hline 4 & - \\
\hline 5 & - \\
\hline 6 & - \\
\hline 7 & - \\
\hline 8 & - \\
\hline 9 & \\
\hline
\end{tabular}

\begin{tabular}{|c|c|}
\hline $\mathbf{N}$ & $\mathbf{P}_{3}$ \\
\hline 10 & $\mathbf{F}_{3}$ \\
\hline 11 & - \\
\hline 12 & - \\
\hline 13 & - \\
\hline 14 & - \\
\hline 15 & $\mathbf{F}_{3}$ \\
\hline 16 & - \\
\hline 17 & - \\
\hline 18 & - \\
\hline 19 & - \\
\hline 20 & $\mathbf{F}_{3}$ \\
\hline 21 & - \\
\hline 22 & - \\
\hline 23 & - \\
\hline 24 & - \\
\hline 25 & $\mathbf{F}_{3}$ \\
\hline 26 & - \\
\hline 27 & - \\
\hline 28 & - \\
\hline 29 & - \\
\hline 30 & $\mathbf{F}_{3}$ \\
\hline
\end{tabular}

En esta tabla se observa que 7 es simétrico con 23, 11 es simétrico con 19 y 13 es simétrico con 17, también se ve que 29 no es simétrico con 1 porque 1 no es primo. Igualmente se observa que $1 \operatorname{sim}_{3}$ llega hasta $P_{n} \#=P_{3} \#=30$.

Teorema 2.20. Si a $X P_{n} \#$ se le resta los números primos determinados por $S_{\mathrm{n}}$ mayores a $P_{\mathrm{n}}$ hasta $P_{\mathrm{k}}$ tomados de menor a mayor, tal que $X P_{n} \#-P_{k}=P_{n+1}+\left((X-1) P_{n} \#\right)$, el resultado será los números primos de $S_{\mathrm{n}}$ tomados de mayor a menor hasta $P_{\mathrm{n}+1}$. 


\section{Demostración.}

En $X P_{n} \#-P_{k}=P_{n+1}+\left((X-1) P_{n} \#\right)$ se ha sumado en el segundo miembro $\left((X-1) P_{n} \#\right)$ por las repeticiones de $\mathrm{C}_{n}$, pero si se considera una sola secuencia, entonces $P_{n} \#-P_{k}=P_{n+1}$.

Ahora sea todo $\mathrm{P}_{\mathrm{O}}$ con $\mathrm{n}<\mathrm{Q} \leq_{\mathrm{k}}$, de manera que a la expresión $P_{n} \#-P_{Q}$ no la dividirá ningún primo $\mathrm{P}_{\mathrm{j}}$ con $\mathrm{j} \leq \mathrm{n}$, por eso $P_{n} \#-P_{O}$ es un numero primo para $S_{n}$.

Ahora $\mathrm{Q}$ debe tomar valores hasta $\mathrm{K}$ tal que $P_{n} \#-P_{k}=P_{n+1}$, ya que si $P_{n} \#-P_{k}$ es igual a algún numero anterior a $P_{n+1}$, este resultado será divisible o igual a algún numero primo anterior a $P_{n+1}$.

Teorema 2.21. En xsim no se tienen en cuenta los números primos anteriores a $P_{\mathrm{n}+1}$ ni sus múltiplos, en $1 \operatorname{sim}_{\mathrm{n}}$ no se tiene en cuenta $0+1$ ni $P_{n} \#-1$, pero en $x_{\text {sim }}$ con $x>1$ si se tiene en cuenta $(x-1) P_{n} \#+1, y$ $x P_{n} \#-1$.

Demostración. La simetría de $P_{n} \#$ se construye con $S_{n}$, y no se tienen en cuenta los números primos anteriores a $\mathrm{P}_{\mathrm{n}+1}$ ni sus múltiplos porque en $S_{n}$ los múltiplos de estos primos ya no aparecen como primos. Ahora en $1 \operatorname{sim}_{\mathrm{n}}$ que es la simetría entre 0 y $P_{n} \#$ no se tiene en cuenta $P_{n} \#-1$, ya que $0+1=1$ no es un numero primo, pero en $x \operatorname{sim}_{\mathrm{n}}$ con $\mathrm{x}>1$ que es la simetría entre $(x-1) P_{n} \#$ y $x P_{n} \#$, si se tiene en cuenta $(x-1) P_{n} \#+1$, y $x P_{n} \#-1$ ya que por el Teorema 2.13 ambos si se consideran números primos en $S_{n}$.

Teorema 2.22. La cantidad de primos que contiene cada una de las dos secciones de 1sim es $\frac{Q_{n-1}\left(P_{n}-1\right)}{2}-1, y$ de $x \operatorname{sim}_{\mathrm{n}}$ con $x>1$ es $\frac{Q_{n-1}\left(P_{n}-1\right)}{2}$.

Demostración. La cantidad de primos que contiene $1 \operatorname{sim}_{\mathrm{n}}$ es $Q_{n-1}\left(P_{n}-1\right)-2$, ya que por el Teorema 2.14. la cantidad de distancias, y por ende la cantidad de primos en $\mathrm{C}_{\mathrm{n}}$ desde $\mathrm{P}_{\mathrm{n}+1}$ (sin incluir a $\left.\mathrm{P}_{\mathrm{n}+1}\right)$ hasta $P_{n} \#+P_{n+1}$ es $Q_{n}=Q_{n-1}\left(P_{n}-1\right)$, pero como $1 \operatorname{sim}_{\mathrm{n}}$ si incluye a $P_{n+1}$, pero no incluye a $P_{n} \#-1$, ni a $P_{n} \#+1$ y $P_{n} \#+P_{n+1}$ porque estos últimos dos pertenecen a $2 \operatorname{sim}_{\mathrm{n}}$, entonces la cantidad de primos que contiene $1 \operatorname{sim}_{\mathrm{n}}$ es $Q_{n-1}\left(P_{n}-1\right)-2$ , ahora como $P_{n} \#$ es par ya que incluye al número primo par 2 como factor, si $P_{n} \#$ se divide en 2 , se obtendrán dos secciones tal que la primera es simétrica con la segunda, de manera que la mitad de cada simetría, es decir, el punto que divide la simetría en dos secciones es frecuencia de todos los primos de $P_{n} \#$ a excepción del 2 , y cada sección en $1 \operatorname{sim}_{\mathrm{n}}$ tendrá $\frac{Q_{n-1}\left(P_{n}-1\right)-2}{2}=\frac{Q_{n-1}\left(P_{n}-1\right)}{2}-1$ primos ; y la cantidad de primos que contiene $x \operatorname{sim}_{n}$ con $\mathrm{x}>1$ es $Q_{n-1}\left(P_{n}-1\right)$, ya que por el Teorema 2.14: $Q_{n}=Q_{n-1}\left(P_{n}-1\right)$ sin incluir a 1 ni a $P_{n+1}$, pero $s i$ incluye a $P_{n} \#-1, P_{n} \#+1$ y $P_{n} \#+P_{n+1}$ , ahora $x \operatorname{sim}_{\mathrm{n}}$ que representa la simetría en las dos secciones que están entre $(x-1) P_{n} \#$, y $x P_{n} \#$, no incluye a $x P_{n} \#+1$ ni a $x P_{n} \#+P_{n+1}$, pero si incluye a $(x-1) P_{n} \#+1,(x-1) P_{n} \#+P_{n+1}$ y a $x P_{n} \#-1$, y cada sección en $x \operatorname{sim}_{\mathrm{n}}$ tendrá $\frac{Q_{n-1}\left(P_{n}-1\right)}{2}$ primos.

Teorema 2.23. En términos generales

$$
(x-1) P_{n+\frac{Q_{n-1}\left(P_{n}-1\right)-2}{2}-(t-1)}=
$$

$x P_{n} \#-P_{n+\frac{Q_{n-1}\left(P_{n}-1\right)-2}{2}+t}, y$

$$
(x-1) P_{n+\frac{Q_{n-1}\left(P_{n}-1\right)-2}{2}}+t=
$$

$$
\begin{aligned}
& x P_{n} \#-P_{n+\frac{Q_{n-1}\left(P_{n}-1\right)-2}{2}-(t-1)}, \text { donde } \\
& t \leq \frac{Q_{n-1}\left(P_{n}-1\right)-2}{2} .
\end{aligned}
$$

Demostración. Por el Teorema 2.20 se sabe que si a $X P_{n} \#$ se le resta los números primos determinados por $S_{n}$ mayores a $P_{n}$ hasta $P_{k}$ tomados de menor a mayor, tal que $X P_{n} \#-P_{k}=P_{n+1}+\left((X-1) P_{n} \#\right)$, el resultado será los números primos de $\mathrm{S}_{\mathrm{n}}$ tomados de mayor a menor hasta $\mathrm{P}_{\mathrm{n}+1}$, de manera que esto es 
una simetría, donde
$\lim _{\mathrm{n}}$, y para $_{\operatorname{xim}}$.

Así para $1 \operatorname{sim}_{\mathrm{n}} \quad 0+P_{n+1}$ es simétrico con $P_{n} \#-P_{n+1}, \quad 0+P_{n+2}$ es simétrico con $P_{n} \#-P_{n+2}$, y $0+P_{n+\frac{Q_{n-1}\left(P_{n}-1\right)-2}{n}}$ es simétrico con $P_{n} \#-P_{n+\frac{Q_{n-1}\left(P_{n}-1\right)-2}{2}}$.

Y para $\operatorname{xsim}_{\mathrm{n}}(x-1) P_{n} \#+1$ es simétrico con $x P_{n} \#-1, \quad(x-1) P_{n} \#+P_{n+1}$ es simétrico con $x P_{n} \#-P_{n+1}, \quad(x-1) P_{n} \#+P_{n+2}$ es simétrico con $x P_{n} \#-P_{n+2}, \mathrm{y}(x-1) P_{n} \#+P_{n+\frac{Q_{n-1}\left(P_{n}-1\right)-2}{2}}$ es simétrico con $x P_{n} \#-P_{n+\frac{Q_{n-1}\left(P_{n}-1\right)-2}{2}}$, pero estos números simétricos son precisamente los números primos determinados en la sección entre $(x-1) P_{n} \#$ y $x P_{n} \#$ por $S_{n}$.

Y se tiene necesariamente que por ejemplo

$$
\begin{aligned}
& (x-1) P_{n+\frac{Q_{n-1}\left(P_{n}-1\right)-2}{2}+1}= \\
& x P_{n} \#-P_{n+\frac{Q_{n-1}\left(P_{n}-1\right)-2}{2}}
\end{aligned}
$$

Porque $(x-1) P_{n+\frac{Q_{n-1}\left(P_{n}-1\right)-2}{2}}$ es el último primo de menor a mayor de la primera sección, de manera que su número primo siguiente es $(x-1) P_{n+\frac{Q_{n-1}}{\left(P_{n}-1\right)-2}}+1$, pero necesariamente el primo siguiente al último primo de la primera sección, es el último primo de mayor a menor de la segunda sección, que es $x P_{n} \#-P_{n+\frac{Q_{n-1}\left(P_{n}-1\right)-2}{2}}$.

Y así mismo $P_{n+\frac{Q_{n-1}\left(P_{n}-1\right)-2}{2}}=$ $x P_{n} \#-P_{n+\frac{Q_{n-1}\left(P_{n}-1\right)-2}{2}+1}$, y en términos generales: $(x-1) P_{n+\frac{Q_{n-1}\left(P_{n}-1\right)-2}{2}-(t-1)}=$

$$
x P_{n} \#-P_{n+\frac{Q_{n-1}\left(P_{n}-1\right)-2}{2}+t}, \mathrm{y}
$$

$$
(x-1) P_{n+\frac{Q_{n-1}\left(P_{n}-1\right)-2}{2}+t}=
$$

$x P_{n} \#-P_{n+\frac{Q_{n-1}\left(P_{n}-1\right)-2}{2}-(t-1)}$, donde

$t \leq \frac{Q_{n-1}\left(P_{n}-1\right)-2}{2}$, ya que no debe salirse del

rango entre $(x-1) P_{n} \#$ y $x P_{n} \#$.

Teorema 2.24. Respecto a los bloques de secuencia $b_{k} \in S_{n}$ se tiene que:

La cantidad o número de bloques en $C_{\mathrm{n}}$ para $n>1$, es igual a la cantidad de productos de fusión $R_{\mathrm{n}}=Q_{\mathrm{n}-1}$.

$$
\sum_{k=1}^{Q_{n-1}} b_{k}=C_{n}=P_{n} \#
$$

\section{Demostración.}

Se sabe por la Demostración 2.7 que bloque de secuencia $\mathbf{b}_{k}$ es la suma de las distancias de una secuencia de un sistema desde $\mathrm{D}_{1}$ o desde la distancia siguiente a la distancia en que termina el bloque anterior, hasta aquella distancia siguiente que es una fusión de dos distancias del sistema inmediatamente anterior, y se sabe por el Teorema 2.18. que la primera distancia $\mathrm{D}_{1} \in \mathrm{C}_{\mathrm{n}}$ para $\mathrm{n}>1$, no es una fusión, y por el Teorema 2.19. se sabe que necesariamente la última distancia $D_{k}$ $\in \mathrm{C}_{\mathrm{n}}$ es una fusión, y como las distancias fusionadas es por los productos de fusión, la cantidad de distancias fusionadas es igual a la cantidad de productos de fusión $\mathrm{R}_{\mathrm{n}}$ por Demostración.

Todos los $b_{k}$ en $\mathrm{C}_{\mathrm{n}}$ están compuestos por todas las $D_{k} \in C_{n}$ empezando desde $D_{1}$ hasta la última distancia $\mathrm{D}_{\mathrm{k}} \in \mathrm{C}_{\mathrm{n}}$, y por el Teorema 2.2. y 2.14.

$$
\sum_{k=1}^{Q_{n}} D_{k}=C_{n}=P_{n} \#
$$

Teorema 2.25. $P_{n} \#>P_{n+1}^{2}$ Para $n>3$, es decir, desde $P_{4}=7$. 
Demostración. Considérese postulado de Bertrand: $\mathrm{n}<\mathrm{P}_{\mathrm{k}} \leq 2 \mathrm{n}$.

Ahora sea $\mathrm{n}=\mathrm{P}_{\mathrm{n}}$ y $\mathrm{P}_{\mathrm{k}}=\mathrm{P}_{\mathrm{n}+1}$, así $\mathrm{P}_{\mathrm{n}}<\mathrm{P}_{\mathrm{n}+1} \leq \mathrm{P}_{\mathrm{n}}$, ahora supóngase el valor máximo de $\mathrm{P}_{\mathrm{n}+1}$ tal que $\mathrm{P}_{\mathrm{n}+1}$ $=2 \mathrm{P}_{\mathrm{n}}$, de manera que $P_{n+1}^{2}=4 P_{n}^{2}$, ahora supóngase cierto que $P_{n} \#>P_{n+1}^{2}$, de manera que también $P_{n} \#>4 P_{n}^{2}$, ahora si se divide esta última expresión en $\mathrm{P}_{\mathrm{n}}$, el resultado es $P_{n-1} \#>4 P_{n}$, y por el mismo postulado de Bertrand $\mathrm{P}_{\mathrm{n}-1}<\mathrm{P}_{\mathrm{n}} \leq 2 \mathrm{P}_{\mathrm{n}-1}$, désele a $\mathrm{P}_{\mathrm{n}}$ el máximo valor tal que $\mathrm{P}_{\mathrm{n}}=2 \mathrm{P}_{n-1}$, así la inecuación $P_{n-1} \#>4 P_{n}$ equivale a $P_{n-1} \#>4\left(2 P_{n-1}\right)$, y si esta expresión se divide en $P_{n-1}$, el resultado es $P_{n-2} \#>8$, esto quiere decir que $P_{n} \#>P_{n+1}^{2}$ si $P_{n-2} \#>8$, y $P_{n-2} \#>8$ para $n>4$, es decir que $P_{n} \#>P_{n+1}^{2}$ para $\mathrm{n}>4$, pero haciendo comprobación numérica $P_{n} \#>P_{n+1}^{2}$ Para $\mathrm{n}>3$, esta diferencia se debe a que en la Demostración se tomaron los casos extremos de que $\mathrm{P}_{\mathrm{n}+1}=2 \mathrm{P}_{n}$ y $\mathrm{P}_{\mathrm{n}}=2 \mathrm{P}_{\mathrm{n}-1}$.

Teorema 2.26. Todo $S_{n}$ con $n>3$ tiene defecto de secuencia desde el primer $C_{\mathrm{n}}$.

Demostración. Todo sistema $S_{n}$ tomando solo un valor de $\mathrm{C}_{\mathrm{n}}$, es decir, sin considerar las demás repeticiones de su patrón, determinara números primos sumando sus distancias a $P_{n+1}$ hasta llegar a $C_{n}=P_{n} \#$ :

$P_{n+1}+\sum_{k=1}^{Q_{n-1}\left(P_{n}-1\right)} D_{k}=P_{n+1}+C_{n}=P_{n+1}+P_{n}^{\#}$

Pero si $P_{n+1}+P_{n} \#>P_{n+1}^{2}$, existirá en $S_{n}$ el defecto de secuencia según la Demostración 2.5, y por el Teorema 2.25. $P_{n} \#>P_{n+1}^{2}$ Para $\mathrm{n}>3$, lo que implica también que $P_{n} \#+P_{n+1}>P_{n+1}^{2}$ Para $\mathrm{n}>3$, ya que para $n \leq 3, P_{n} \#+P_{n+1}<P_{n+1}^{2}$.

Teorema 2.27. No todos los números de Euclides de la forma $P_{n} \#+1$ son números primos.

Demostración. En la Demostración del Teorema 2.6 se mostró que para $\mathrm{S}_{\mathrm{n}}, \mathrm{x}\left(P_{n} \#\right)+1$ será un numero primo, sea x=1, y por el Teorema $2.8, S_{n}$ será correcta en la determinación de números primos hasta llegar a $\left(P_{n+1}\right)^{2}$, de ahí en adelante el sistema $S_{n}$ determinará tanto números primos como números compuestos, y por el Teorema 2.26 todo $S_{n}$ con $n>3$ tiene defecto de secuencia, por eso los números de EUCLIDES de la forma $P_{n} \#+1$ para n $>3$ están en la zona de defecto de secuencia de $S_{n}$, lo que implica que $P_{n} \#+1$ puede ser un numero primo o un numero compuesto.

Teorema 2.28. En $S_{\mathrm{n}}, P_{\mathrm{n}+1}+D_{1}=P_{\mathrm{n}+2}<P_{n+1}^{2}$.

Demostración. Por el Teorema 2.17 se sabe que $\mathrm{P}_{\mathrm{n}+1}+\mathrm{D}_{1}<P_{n}^{2}$, y como evidentemente $P_{n+1}^{2}>P_{n}^{2}$, entonces también $\mathrm{P}_{\mathrm{n}+1}+\mathrm{D}_{1}<P_{n+1}^{2}$.

Teorema 2.29. $P_{n}^{2}>P_{n+1}$.

Demostración. Considérese de nuevo el postulado de Bertrand, de manera que $\mathrm{P}_{\mathrm{n}}<\mathrm{P}_{\mathrm{n}+1} \leq 2 \mathrm{P}_{\mathrm{n}}$, así $\mathrm{P}_{\mathrm{n}+1} \leq$ $2 \mathrm{P}_{\mathrm{n}}$, ahora si se reemplaza en el segundo miembro de la inecuación el numero 2 por $\mathrm{P}_{\mathrm{n}}$, entonces si $\mathrm{P}_{\mathrm{n}+1} \leq$ $2 \mathrm{P}_{\mathrm{n}}$, entonces $\mathrm{P}_{\mathrm{n}+1}<\mathrm{P}_{\mathrm{n}} * \mathrm{P}_{\mathrm{n}}=P_{n}^{2}$ para $\mathrm{P}_{\mathrm{n}}>2$, pero por comprobación numérica se sabe que también se cumple $P_{n}^{2}>P_{n+1}$ para $\mathrm{P}_{\mathrm{n}}=2$.

Teorema 2.30. Siempre entre $P_{\mathrm{n}}$ y $P_{n}^{2}$ existirá por lo menos $N$ números primos, de manera que $2^{N} \leq P_{n}, y$

$$
N=\left[\frac{\log P_{n}}{\log 2}\right] .
$$

Demostración. Por el postulado de Bertrand, se tiene que:

$$
\mathrm{P}_{\mathrm{n}}<\mathrm{P}_{k_{1}}<2 \mathrm{P}_{\mathrm{n}}
$$

De manera que: $\left(2 \mathrm{P}_{\mathrm{n}}\right)<P_{k_{2}}<\left(4 \mathrm{P}_{\mathrm{n}}\right), \quad\left(4 \mathrm{P}_{\mathrm{n}}\right)<P_{k_{3}}$ $<\left(8 \mathrm{P}_{\mathrm{n}}\right),\left(8 \mathrm{P}_{\mathrm{n}}\right)<P_{k_{4}}<\left(16 \mathrm{P}_{\mathrm{n}}\right), \ldots \ldots \ldots,\left(2^{\mathrm{N}-1}\right) \mathrm{P}_{\mathrm{n}}<P_{k_{N}}<\left(2^{\mathrm{N}}\right) \mathrm{P}_{\mathrm{n}}$

$$
\text { Así }\left(2^{\mathrm{N}}\right) \mathrm{P}_{\mathrm{n}} \leq P_{n}^{2} \text { si } 2^{N} \leq P_{n} .
$$

Ahora si se desea conocer el valor de $\mathrm{N}$ conociendo $P_{n}$, en la expresión $2^{N} \leq P_{n}$ se despeja $\mathrm{N}$, de manera que $\log 2^{N} \leq \log P_{n}, \mathrm{~N} \log 2 \leq \log P_{n}$, y despejando a $\mathrm{N}$ : 


$$
N \leq \frac{\log P_{n}}{\log 2}
$$

Ahora $\mathrm{N}$ toma el valor entero que no supere a $\frac{\log P_{n}}{\log 2}$, ahora sea $[\mathrm{x}]$ la función máximo entero menor o igual a x, de manera que $N=\left[\frac{\log P_{n}}{\log 2}\right]$.

Teorema 2.31. En $S_{\mathrm{n}}$ existen por lo menos dos productos de fusión antes de $P_{n+1}^{2}$, es decir, antes del defecto de secuencia.

Demostración. Recuérdese en la Demostración 2.6 que los productos de fusión en $S_{n}$ son los productos de $\mathrm{P}_{\mathrm{n}}$ con los primos determinados con las distancias de la secuencia $\mathrm{C}_{\mathrm{n}-1}$, es decir, con los números que son diferentes a los primos anteriores a $\mathrm{P}_{\mathrm{n}} \mathrm{o}$ a sus múltiplos, $\mathrm{y}$ los dos primeros números diferentes a los primos anteriores a $\mathrm{P}_{\mathrm{n}}$, son el mismo $\mathrm{P}_{\mathrm{n}}$ y $\mathrm{P}_{\mathrm{n}+1}$, de manera que los primeros productos de fusión son $\mathrm{P}_{\mathrm{n}} * \mathrm{P}_{\mathrm{n}}$ y $\mathrm{P}_{\mathrm{n}} * \mathrm{P}_{\mathrm{n}+1}, \mathrm{y}$ como $\mathrm{P}_{\mathrm{n}}<\mathrm{P}_{\mathrm{n}+1}$, con certeza $\mathrm{P}_{\mathrm{n}} * \mathrm{P}_{\mathrm{n}}<\mathrm{P}_{n+1}^{2}$ y $\mathrm{P}_{\mathrm{n}}{ }^{*} \mathrm{P}_{\mathrm{n}+1}<$ $P_{n+1}^{2}$.

Teorema 2.32. En $C_{\mathrm{n}}$ existen por lo menos dos distancias de fusión $D_{\mathrm{h}}$ y $D_{\mathrm{k}^{\prime}}$ donde $k>h$, antes de $P_{n+1}^{2}$, es decir, antes del defecto de secuencia, si $P_{n+1}+\left(\sum_{j=1}^{k} D_{j}\right)<P_{n+1}^{2}$

cias $D_{\mathrm{h}}$, si $P_{n+1}+\left(\sum_{j=1}^{k} D_{j}\right)=P_{n+1}^{2}$.

Demostración. Por el Teorema 2.31. se sabe que en $S_{n}$ existen por lo menos dos productos de fusión antes de $P_{n+1}^{2}$, y estos dos productos de fusión crean en $\mathrm{C}_{\mathrm{n}}$ dos distancias de fusión, pero si

$$
P_{n+1}+\left(\sum_{j=1}^{k} D_{j}\right)=P_{n+1}^{2}
$$

, donde $\mathrm{D}_{\mathrm{k}}$ es la última distancia de fusión, quiere decir que su límite superior es $P_{n+1}^{2}$, pero $P_{n+1}^{2}$ es el primer producto de fusión de defecto de secuencia, así $\mathrm{D}_{k}$ es en realidad $D_{k_{1}}$, y su límite superior real será superior a $P_{n+1}^{2}$

$$
P_{n+1}+\left(\sum_{j=1}^{k_{1}} D_{j}\right)>P_{n+1}^{2} \text {, por ello en este }
$$

, así , por ello en este tancia de fusión $\mathrm{D}_{\mathrm{h}}$ antes de $P_{n+1}^{2}$, de manera que $P_{n+1}+\left(\sum_{j=1}^{h} D_{j}\right)<P_{n+1}^{2}$.

Teorema 2.33. La cantidad de productos de fusión en $C_{\mathrm{n}}$ antes de $P_{n+1}^{2}$, será igual a $k+1$, de manera que $P_{n}\left(\sum_{j=1}^{k} D_{j}\right)<2 D_{1} P_{n}+D_{1}^{2}$, para $D_{j} \in C_{n-1}$.

Demostración. Sea todo $D_{j} \in C_{n-1}$, así en vez de $P_{n+1}=P_{n}+D_{0}$ con $D_{0} \in C_{n}$, será $P_{n+1}=P_{n}+D_{1} \quad$ con $\quad D_{1} \in C_{n-1}$, ahora $P_{n+1}^{2}=\left(P_{n}+D_{1}\right)^{2}=P_{n}^{2}+2 D_{1} P_{n}+D_{1}^{2}$, ahora sea $P_{n+k}=P_{n}+\sum_{j=1}^{k} D_{j}$, así:

$$
P_{n} P_{n+k}=P_{n}^{2}+P_{n} \sum_{j=1}^{k} D_{j}
$$

Pero $P_{n} P_{n+k}$ debe ser lo más próximo a $P_{n+1}^{2} \sin$ sobrepasarlo, es decir que $P_{n} P_{n+k}<P_{n+1}^{2}$, que equivale a

$$
P_{n}\left(\sum_{j=1}^{k} D_{j}\right)<2 D_{1} P_{n}+D_{1}^{2}
$$
, y la cantidad de productos de fusión será igual a $\mathrm{k}+1$, ya que se cuentas también el producto de fusión $P_{n}^{2}$.

Teorema 2.34. La cantidad de distancias de fusión en $C_{\mathrm{n}}$ antes de $P_{n+1}^{2}$, será igual a $k+1$, de manera que $P_{n}\left(\sum_{j=1}^{k} D_{j}\right)<2 D_{1} P_{n}+D_{1}^{2}$, 


$$
\begin{aligned}
& P_{n+1}+\left(\sum_{j=1}^{k} D_{j}\right)<P_{n+1}^{2}, \text { y será } k s i \\
& P_{n+1}+\left(\sum_{j=1}^{k} D_{j}\right)=P_{n+1, \text { para }}^{2} D_{j} \in C_{n-1} .
\end{aligned}
$$

Demostración. Por el Teorema 2.33. se sabe que la cantidad de productos de fusión en $\mathrm{C}_{\mathrm{n}}$ antes de $P_{n+1}^{2}$, será igual a $\mathrm{k}+1$, de manera que $P_{n}\left(\sum_{j=1}^{k} D_{j}\right)<2 D_{1} P_{n}+D_{1}^{2}$, para $D_{j} \in C_{n-1, y}$ los productos de fusión crean distancias de fusión, pero en el caso de que $P_{n+1}+\left(\sum_{j=1}^{k} D_{j}\right)=P_{n+1}^{2}$, como se explicó en Teorema 2.32., será uno menos, es decir k.

El siguiente Teorema se construye a partir de esta idea: "existen arbitrariamente grande vacíos en la serie de los primos. Dicho de otra manera, dado cualquier entero positivo $\mathrm{k}$, existen $\mathrm{k}$ enteros compuestos consecutivos." (Niven y Zukcerman 1969, 23).

Teorema 2.35. Todo número de la forma $P_{n} \# \mp k$, con $1<k \leq P_{n}$ no es número primo.

Demostración. Todo numero $k \leq P_{n}$, es un numero primo o un numero compuesto por los primos anteriores a $P_{n}$, así la expresión $P_{n} \# \mp k$ es divisible por algún o algunos primos anteriores o igual a $P_{n}$, pero k>1, ya que $P_{n} \# \mp 1$ no es divisible por los primos anteriores o igual a $P_{n}$.

Teorema 2.36. En $S_{\mathrm{n}}$ la distancia máxima max $D_{k} \in C_{n}$ es $2\left(f_{n+1}\right) \geq \max D_{k} \geq P_{n+1}-1$, sin tener en cuenta el defecto de secuencia, donde $f_{n+1}$ representa el n+1-ésimo numero en la sucesión de Fibonacci.

Demostración. Por el Teorema 2.35 se sabe que todo número de la forma $P_{n} \# \mp k$, con $1<k \leq P_{n}$ no es primo, pero para $S_{n}$, el número de la forma
$P_{n} \# \mp P_{n+1}$ si es primo, ya que no es divisible por los primos anteriores o igual a $P_{n}$, y se sabe también que para $S_{\mathrm{n}}, P_{n} \# \mp 1$ también es primo, y la diferencia absoluta $\left|\left(P_{n} \# \mp P_{n+1}\right)-\left(P_{n} \# \mp 1\right)\right|=P_{n+1}-1$, así la distancia máxima de $S_{n}$ no puede ser menor a $P_{n+1}-1$.

Ahora $\max D_{k} \in C_{1}=2, \quad \max D_{k} \in C_{2}=4$, $\max D_{k} \in C_{3}=6$ y $\max D_{k} \in C_{4}=10$, ahora hágase la suposición extrema de que todo $\max D_{k} \in C_{n}$ $=\left(\max D_{k} \in C_{n-1}\right)+\left(\max D_{k} \in C_{n-2}\right)$.

Sea $\max D_{k} \in C_{1}=2\left(f_{2}\right), \max$ $D_{k} \in C_{2}=2\left(f_{3}\right), \max D_{k} \in C_{3}=2\left(f_{4}\right)$ y $\max$ $D_{k} \in C_{n}=2\left(f_{n+1}\right)$, donde $f_{2}=1$ es el segundo número en la sucesión de Fibonacci, $f_{3}=2$ es el tercer número, $f_{4}=3$ es el cuarto número, y así sucesivamente, y como a max $D_{k} \in C_{n}$ se le está dando un valor máximo extremo de $2\left(f_{n+1}\right)$, el verdadero valor de $\max D_{k} \in C_{n}$ no puede superar

o ser mayor que $2\left(f_{n+1}\right)$, por ello $2\left(f_{n+1}\right) \geq \max$ $D_{k} \geq P_{n+1}-1$.

Teorema 2.37. Sea $C_{\mathrm{n}}$, con $n>4$, es decir, con defecto de secuencia, llámese $U_{\mathrm{n}}=P_{n+1}^{2}-P_{n+1}$ la parte util de $C_{\mathrm{n}}$ antes del defecto de secuencia, de manera que $\lim _{n \rightarrow \infty} \frac{U_{n}}{C_{n}}=0$.

Demostración. Supóngase que $\lim _{n \rightarrow \infty} \frac{U_{n}}{C_{n}}=0$ es cierto, de manera que $\mathrm{C}_{\mathrm{n}}>\mathrm{U}_{\mathrm{n}}$, ahora si a ambos lados de esta inecuación se le suma un valor se mantiene la inecuación, tal que $\mathrm{C}_{\mathrm{n}}+P_{n+1}>\mathrm{U}_{\mathrm{n}}+P_{n+1}$.

Ahora sea $\mathrm{U}_{\mathrm{n}}=P_{n+1}^{2}-P_{n+1}$ y $\mathrm{C}_{\mathrm{n}}=P_{n} \#$, si a ambas ecuaciones se les suma $P_{n+1}$, se tiene que:

$$
\lim _{n \rightarrow \infty} \frac{U_{n}}{C_{n}}=\frac{P_{n+1}^{2}-P_{n+1}}{P_{n} \#} \approx \frac{P_{n+1}^{2}}{P_{n} \#+P_{n+1}}
$$

Ahora tómese esta última expresión e inviértase así: 


$$
\frac{P_{n} \#+P_{n+1}}{P_{n+1}^{2}}=\frac{P_{n} \#}{P_{n+1}^{2}}+\frac{P_{n+1}}{P_{n+1}^{2}}=\frac{P_{n} \#}{P_{n+1}^{2}}+\frac{1}{P_{n+1}}, \text { ahora }
$$
1

la fracción $\overline{P_{n+1}}$ tiende a cero cuando n aumenta, así que se puede omitir, de manera que solamente queda $\frac{P_{n} \#}{P_{n+1}^{2}}$

que $P_{n+1} \leq 2 P_{n}$, asi $P_{n+1}^{2} \leq 4 P_{n}^{2}$, así el denominador de la fracción se puede reemplazar por su valor máximo así: $\frac{P_{n} \#}{4 P_{n}^{2}}=: \frac{P_{n-1} \#}{4 P_{n}}$, y por el mismo Teorema de Bertrand $P_{n} \leq 2 P_{n-1}$, así el denominador de esta última fracción de nuevo se reemplaza por su valor máximo: $\frac{P_{n-1} \#}{4\left(2 P_{n-1}\right)}=\frac{P_{n-2} \#}{8}$, recuérdese que se le asigno valores máximos al denominador, así los valores reales del denominador pueden tomar valores menores, y si la fracción se invierte: $\frac{8}{P_{n-2} \#}$, y evidentemente esta fracción tiende a cero al crecer $n$, pero como se dijo, al numerador se le ha asignado valores máximos, y puede tomar valores menores, así que la

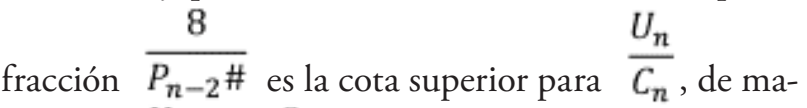

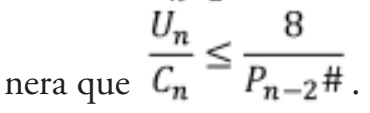

Teorema 2.38. Para todo $S_{n}$ con $n>4$, el defecto de secuencia aparece en la primera sección de 1 sim.

Demostración. Sea $n=5$, así el defecto de secuencia empieza desde $P_{n+1}^{2}=P_{6}^{2}=13^{2}=169$. Ahora la primera sección de $1 \operatorname{sim}_{5}$ está entre 0 y $\frac{P_{5} \#}{2}=1155$, asi $P_{6}^{2}<\frac{P_{5} \#}{2}$, y por el Teorema 2.37., $\mathrm{C}_{\mathrm{n}}=P_{n} \#$ aumenta su proporción con respecto a $U_{n}$ al aumen$\operatorname{tar} \mathrm{n}$, ahora usando un razonamiento similar al de la Demostración del Teorema 2.37. se tiene que $\lim _{n \rightarrow \infty} \frac{P_{n+1}^{2}}{P_{n} \#} \approx \frac{4 P_{n}^{2}}{P_{n} \#} \approx \frac{4 P_{n}}{P_{n-1} \#} \approx \frac{8 P_{n-1}}{P_{n-1} \#} \approx \frac{8}{P_{n-2} \#}=0$ , y como se le dio valores máximos al numerador, se tiene que $\lim _{n \rightarrow \infty} \frac{P_{n+1}^{2}}{P_{n} \#}=0$, de manera que si $P_{n} \#$ aumenta su proporción con respecto a $P_{n+1}^{2}$ al aumentar n, entonces $\frac{P_{n} \#}{2}$ también aumenta su proporción con respecto a $P_{n+1}^{2}$ al aumentar $\mathrm{n}$, y si en $\mathrm{n}=5$, $P_{6}^{2}<\frac{P_{5} \#}{2}$, entonces $P_{n+1}^{2}<\frac{P_{n} \#}{2}$ para todo $\mathrm{n}>4$.

Teorema 2.39. Sea $R_{n}^{\prime}$ la cantidad de productos de fusión de defecto de secuencia en $P_{n} \#+P_{n+1}$, de ma${ }_{\text {neraque }} R_{n}^{\prime}=\sum_{j=1}^{k} \rho\left(x_{j}\right)$, donde $x_{j}=\left[\frac{P_{n} \#+P_{n+1}}{P_{n+j}}\right]$ $k$ toma el valor de $P_{n+k}$ que es el numero primo anterior y más cercano a $\sqrt{P_{n} \#+P_{n+1}}, y \rho\left(x_{j}\right)$ es la función que determina la cantidad de números primos para $S_{n}$ que hay en $x_{j}$ sin incluir a los primos anteriores a $P_{n+j}$.

Demostración. Sea $x_{j}=\left[\frac{P_{n} \#+P_{n+1}}{P_{\ldots} \ldots}\right]$ la cantidad de veces que esta $P_{n+j}$ en $P_{n} \#+P_{n+1}$, pero de $x_{j}$ solo interesa los números primos para $S_{n}$ mayores o iguales a $P_{n+j}$, así la función $\rho\left(x_{j}\right)$ determina esta cantidad de primos que son los que participan en los productos de fusión de defecto de secuencia.

$$
\text { Ahora }\left[\frac{P_{n} \#+P_{n+1}}{P_{n+j}}\right] \geq P_{n+j} \text {, ya que si }
$$

$\left[\frac{P_{n} \#+P_{n+1}}{P_{n+j}}\right]<P_{n+j}$ entonces no habría primos para $S_{n}$ en $x_{j}$ mayores o iguales a $P_{n+j}$.

Ahora $1 \leq j \leq k$, tal que $k$ viene del $P_{n+k}$ que es el primo máximo tal que $\left[\frac{P_{n} \#+P_{n+1}}{P_{n+k}}\right] \geq P_{n+k}$, 
ahora $P_{n} \#+P_{n+1} \geq P_{n+k}^{2}, \mathrm{y} \sqrt{P_{n} \#+P_{n+1}} \geq P_{n+k}$

Bien, los productos de fusión de defecto de secuencia en $P_{n} \#+P_{n+1}$ son de la forma $\left(P_{n+j}^{a_{0}} P_{n+j+1}^{a_{1}} P_{n+j+2}^{a_{2}} \ldots \ldots . P_{n+j+i}^{a_{i}}\right) \leq P_{n} \#+P_{n+1}$, y el producto entre paréntesis es un primo para $S_{n}$ , así que no hay problema por las potencias.

Teorema 2.40. Si la función $\pi(x)$ denota la cantidad de primos en $x$, y si $x=P_{n} \#+P_{n+1}$, entonces:

$\left[\frac{\log \left(P_{n} \#+P_{n+1}\right)}{\log 2}\right] \leq \pi\left(P_{n} \#+P_{n+1}\right) \leq$

$\left(\prod_{k=2}^{n}\left(P_{k}-1\right)\right)+n+1$

Y también:

$\pi\left(P_{n} \#+P_{n+1}\right)=\left(\prod_{k=2}^{n}\left(P_{k}-1\right)\right)+n+1-R_{n}^{\prime}$.

Demostración. Por el Teorema 2.30,

$N=\left[\frac{\log \left(P_{n} \#+P_{n+1}\right)}{\log 2}\right]$, y por el Teorema 2.14.

$Q_{n}=Q_{n-1}\left(P_{n}-1\right)$ у $Q_{n-1}=Q_{n-2}\left(P_{n-1}-1\right)$

Así $Q_{n}=Q_{n-2}\left(P_{n-1}-1\right)\left(P_{n}-1\right)$ y

$Q_{n-2}=Q_{n-3}\left(P_{n-2}-1\right)$

Así $Q_{n}=Q_{n-3}\left(P_{n-2}-1\right)\left(P_{n-1}-1\right)\left(P_{n}-1\right), \mathrm{y}$ en general:

$Q_{n}=\left(P_{2}-1\right)\left(P_{3}-1\right)\left(P_{4}-1\right) \ldots \ldots \ldots \ldots\left(P_{n}-1\right)$

$=\prod_{k=2}^{n}\left(P_{k}-1\right)$.

Ahora las $D_{k} \in C_{n}$ determinan números primos para $S_{n}$, de manera que $Q_{n}$ es la cantidad de primos determinados en $C_{n}$, y como por el Teorema
2.1 las $D_{k}$ empiezan a sumar desde $P_{n+1}$, antes de los primos determinados por $D_{k}$, están los primos $P_{1}, P_{2}, P_{3}, \ldots \ldots \ldots, P_{n+1}$, es decir $\mathrm{n}+1$ primos.

Ahora si $1 \leq n \leq 3$, entonces

$\pi\left(P_{n} \#+P_{n+1}\right)=\left(\prod_{k=2}^{n}\left(P_{k}-1\right)\right)+n+1$.

Si $n>3$, entonces

$\pi\left(P_{n} \#+P_{n+1}\right)<\left(\prod_{k=2}^{n}\left(P_{k}-1\right)\right)+n+1$,

esto es porque por el Teorema 2.26 todo $S_{n}$ con $n>3$ tiene defecto de secuencia, pero si a

$$
\left(\prod_{k=2}^{n}\left(P_{k}-1\right)\right)+n+1 \quad \text { se le resta los falsos }
$$

primos por los productos de fusión de defectos de secuencia cuya cantidad en $P_{n} \#+P_{n+1}$ es $R_{n}^{\prime}$ por el Teorema 2.39, entonces

$$
\pi\left(P_{n} \#+P_{n+1}\right)=\left(\prod_{k=2}^{n}\left(P_{k}-1\right)\right)+n+1-R_{n}^{\prime} \text {. }
$$

Teorema 2.41. Siendo cualquier $x P_{n} \#-\delta$ un númeroprimo, con $x, \delta \in N, y P_{n+1}<\delta<\frac{P_{n} \#}{2}, P_{n+1}^{2}$, entonces $\delta$ es con toda certeza un número primo.

Demostración. Ya se sabe por la simetría secuencial, que los números primos de la primera sección de $x P_{n} \#$, y también de $P_{n} \#$, son simétricos con la segunda sección de $x P_{n} \#$, pero si hay un $x P_{n} \#-\delta$ que sea primo, será primo a pesar del defecto de secuencia, y si $\delta<\frac{P_{n} \#}{2}$, entonces $x P_{n} \#-\delta$ es un número primo que está en la segunda sección de la simetría, y si $\delta<P_{n+1}^{2}$, entonces su número simétrico en la primera sección de $1 \boldsymbol{s i m}_{\mathrm{n}}$ esta antes del defecto de secuencia, y $P_{n+1}<\delta$, ya que si $P_{n+1}=\delta$, es obvio que $P_{n+1}$ es primo. 
Ejemplo 2.1. Sea

$2 P_{n} \#=2 P_{4} \#=2(7 \#)=2(210)=420$

Ahora sea $\delta=83$, y $420-83=337$, y 337 es un

numero primo, y como $83<\frac{P_{4} \#}{2}=105$, y

$83<P_{4+1}^{2}=11^{2}=121$, entonces 83 es con toda certeza un numero primo.

\section{Teorema fundamental de la teoría de la distribu- ción de los números primos}

Formula general de los números primos

En $S_{\mathrm{n}}$, todo número primo mayor a 3 es de la forma:

$$
P_{n+k+1}=\left(P_{n+1}+\gamma P_{n} \#+\sum_{k=1}^{Z} D_{k}\right)<P_{n+1}^{2}
$$

$$
\text { Tal }
$$$$
\text { que }
$$

$$
P_{n+k+1} \in S_{n},
$$

$1 \leq z \leq \prod_{k=2}^{n}\left(P_{k}-1\right), 0 \leq \gamma \leq 3$ donde $D_{k} \in C_{n}$.

Demostración. Por el Teorema 2.1 se sabe que las distancias $D_{k} \in C_{n}$ del sistema $S_{n}$ construido hasta $P_{n}$, se empiezan a sumar desde $P_{n+1}$.

Ahora la expresión $\gamma P_{n} \#$ representa las repeticiones de $\mathrm{C}_{\mathrm{n}}$, y por el Teorema 2.2 se sabe que $C_{n}=P_{n} \#$, y $0 \leq \gamma \leq 3$ ya que por el Teorema 2.26 todo $S_{n}$ con $\mathrm{n}>3$ tiene defecto de secuencia.

En la sumatoria $\sum_{k=1}^{Z} D_{k}, 1 \leq z \leq Q_{n+1}\left(P_{n}-1\right)$, ya que por el Teorema 2.14. La cantidad de distancias $Q_{n}$ existentes en un $C_{n}$, es

$$
Q_{n}=Q_{n-1}\left(P_{n}-1\right)=\prod_{k=2}^{n}\left(P_{k}-1\right) \text {. }
$$

Ahora $\left(P_{n+1}+\gamma P_{n} \#+\sum_{k=1}^{Z} D_{k}\right)<P_{n+1}^{2}$, ya que por el Teorema 2.8. Cada sistema $S_{n}$ funcionará perfectamente en la determinación de números primos hasta llegar al cuadrado del primo $\mathrm{P}_{\mathrm{n}+1}$, siguiente al primo $\mathrm{P}_{\mathrm{n}}$ hasta donde se construyó el sistema.

Y la fórmula de $P_{n+1+k}$ expresa la forma de todo número primo mayor a 3 , ya que 2 no es un $P_{n+1}$, y por lo tanto tampoco un $P_{n+1+k}$, y 3 si es un $P_{n+1}$ pero no un $P_{n+1+k}$.

Esta fórmula de $P_{n+1+k}$ siempre será utilizable, ya que por el Teorema 2.28. $\mathrm{P}_{\mathrm{n}+1}+\mathrm{D}_{1}<P_{n+1}^{2}$.

Sin embargo, para utilizar esta fórmula se deben de construir completamente las distancias de cada secuencia $C_{n} \in S_{n}$, pero el valor de $C_{n}=P_{n} \#$, crece rápidamente al aumentar $\mathrm{n}, \mathrm{y}$ como dice el Teorema

2.15, $Q_{n+1}$ aumenta su proporción con respecto a $Q_{n}$ al crecer el valor de n, de manera que para el cálculo de las distancias $\mathrm{D}_{\mathrm{k}} \in C_{n}$ se necesita la ayuda de una computadora para hacer los cálculos cada vez más extensos.

\section{Otra Demostración del postulado de Bertrand}

Como se ha visto anteriormente, las distancias de cada sistema se determinan con las distancias del sistema anterior, y algunas de ellas se unen por los productos de fusión, ahora considérese el siguiente caso extremo:

En $S_{1}$ los números primos se van determinando así: $3+2+2+2+2+2+2+2 \ldots \ldots \ldots$

Ahora supóngase que en $S_{2}$ los números primos se van determinando así: $3+2+4+4+4+4+4+4+\ldots \ldots$, en 
$S_{3}: 3+2+4+8+8+8+8+\ldots$. y así en general los números primos se van construyendo con la suma a $P_{2}=3$ de las potencias de 2 , así: $3+(2+4+8+16+32+64+\ldots .$.$) .$

Ahora se sabe que

$1+a^{1}+a^{2}+a^{3}+\ldots \ldots+a^{k}=\sum_{n=0}^{k} a^{n}=\frac{1-a^{k+1}}{1-a}$

(Grossman 1996, app 1, A-5).

De esta fórmula se tiene que $\sum_{n=1}^{k} a^{n}=\frac{a^{k+1}-a}{a-1}$, ahora si $a=2$, entonces $\sum_{n=1}^{k} 2^{n}=2^{k+1}-2$ $\mathrm{y} \frac{\left(\sum_{n=1}^{k} 2^{n}\right)}{2}+1=\frac{2^{k+1}-2}{2}+1=2^{k}$, así $\sum_{n=1}^{k} 2^{n}-\frac{\left(\sum_{n=1}^{k} 2^{n}\right)}{2}-1=2^{k+1}-2-2^{k}=$ $2 * 2^{k}-2^{k}-2=2^{k}(2-1)-2=2^{k}-2=$ $\sum_{n=1}^{k-1} 2^{n}$. Ahora sea $\beta=\sum_{n=1}^{k} 2^{n}$, así que $3+\beta=P_{r}$, y $3+\beta-\frac{\beta}{2}-1=3+\frac{\beta}{2}-1=P_{r-1}$ ahora $2 P_{r-1}=6+\beta-2=4+\beta$, y $4+\beta-1=3+\beta=2 P_{r-1}-1=P_{r}$, así $P_{r-1}<P_{r}=2 P_{r-1}-1, P_{r-1}<P_{r}<2 P_{r-1}$.

Ahora sea $0<\delta<\left(P_{r}-P_{r-1}\right)$ con $\delta \in+\mathbf{R}$, así se cumple la desigualdad

$P_{r-1}+\delta<P_{r}<2 P_{r-1}+2 \delta$, y si $\delta=\left(P_{r}-P_{r-1}\right)$, entonces $P_{r-1}+\delta=P_{r}$, y $P_{r}<P_{r+1}<2 P_{r}$.

Así se concluye que si $\mathrm{n}$ es un número real positivo mayor a 1 , se tiene que $n<P<2 n$.

\section{Conclusion}

La distribución de los números primos parece aleatoria por la aparición aparentemente caótica de sus distancias, pero en realidad su distribución es sistemática, como se observó en la construcción de los sistemas de distribución y sus secuencias, pero a medida que se va determinando un nuevo número primo, este afecta la distribución y se crea un nuevo sistema con una nueva secuencia que cambia la manera de predecir números primos, y así los sistemas se van volviendo más complejos, con secuencias más extensas y distancias más variadas, así que los números primos presentan, como se puede llamar, una distribución compleja.

\section{Bibliografía}

Apostol, TM (1980), Introducción a la teoría analitica de números, edición en español, Editorial Reverte S.A., Barcelona.

Caro, VE (1937), Los números, Editorial Minerva S.A., Bogotá.

Grossman, S (1996), Algebra lineal. Quinta edición, McGrawHill, Bogotá.

Jiménez, LR, Gordillo, JE \& Rubiano, GN (2004), Teoría de números [para principiantes], segunda edición, Universidad Nacional de Colombia, Bogotá.

Mattson, HF (1993), Discrete Mathematics, John Wiley \& Sons Inc., New Jersey.

Mora, W (2014), Introducción a la teoría de números. Ejemplos y algoritmos, primera edición, Revista digital matemática, educación e internet, Recuperado de: https://repositoriotec.tec.ac.cr/bitstream/ handle/2238/6299/introducci\%C3\%B3n-teor\%C3\%ADa$\mathrm{n} \% \mathrm{C} 3 \%$ BAmeros.pdf?sequence $=1$ \&isAllowed $=\mathrm{y}$

Niven, I, Zuckerman, HS (1969). Introducción a la teoría de números, Limusa-Wiley, México.

Ross, KA., Wright, CR (1990), Matemáticas discretas, segunda edición, Prentice-Hall Hispanoamericana S.A., México.

Vinogradov, I (1977), Fundamentos de la teoría de los números, segunda edición, Editorial MIR, Moscú.

Vorobiov, NN (1974). Números de Fibonacci, Editorial MIR, Moscú. 
